\title{
NHEG mechanics: laws of near horizon extremal geometry (thermo)dynamics
}

\author{
K. Hajian, ${ }^{a, b}$ A. Seraj ${ }^{a}$ and M.M. Sheikh-Jabbari ${ }^{a}$ \\ ${ }^{a}$ School of Physics, Institute for Research in Fundamental Sciences (IPM), \\ P.O. Box 19395-5531, Tehran, Iran \\ ${ }^{b}$ Department of Physics, Sharif University of Technology, \\ P.O. Box 11155-9161, Tehran, Iran \\ E-mail: kamalhajian@physics.sharif.edu, ali_seraj@ipm.ir, \\ jabbari@theory.ipm.ac.ir
}

Abstract: Near Horizon Extremal Geometries (NHEG) are solutions to gravity theories with $\mathrm{SL}(2, \mathbb{R}) \times \mathrm{U}(1)^{N}$ (for some $N$ ) symmetry, are smooth geometries and have no event horizon, unlike black holes. Following the ideas by R. M. Wald, we derive laws of NHEG dynamics, the analogs of laws of black hole dynamics for the NHEG. Despite the absence of horizon in the NHEG, one may associate an entropy to the NHEG, as a Noether-Wald conserved charge. We work out "entropy" and "entropy perturbation" laws, which are respectively universal relations between conserved Noether charges corresponding to the NHEG and a system probing the NHEG. Our entropy law is closely related to Sen's entropy function. We also discuss whether the laws of NHEG dynamics can be obtained from the laws of black hole thermodynamics in the extremal limit.

Keywords: Black Holes, Space-Time Symmetries, Classical Theories of Gravity, Gauge Symmetry

ARXIV EPRINT: 1310.3727 


\section{Contents}

1 Introduction 1

2 Near Horizon Extremal Geometries (NHEG) 3

2.1 Extremal horizons and near horizon limits 4

2.2 Relation between $\mathrm{SL}(2, \mathbb{R})$ and $\mathrm{U}(1)$ generators 5

3 NHEG conserved charges $\quad 6$

3.1 Noether charge density of non-Abelian symmetries 6

$3.2 \mathrm{SL}(2, \mathbb{R})$ conserved charges 8

3.3 NHEG entropy as a conserved charge $\quad 9$

4 Laws of NHEG dynamics $\quad 10$

4.1 Zeroth law of NHEG dynamics 11

$\begin{array}{lll}4.2 & \text { NHEG entropy law } & 11\end{array}$

$\begin{array}{lll}4.3 & \text { NHEG entropy perturbation law } & 13\end{array}$

5 NHEG vs. extremal black hole $\quad 17$

$\begin{array}{lll}5.1 & \text { Near horizon limit of extremal black holes } & 18\end{array}$

$\begin{array}{ll}5.2 & \text { NHEG entropy perturbation law and near horizon limit } \\ 5.3 & 19\end{array}$

$\begin{array}{lll}5.3 & \text { Interpretation of } k^{i}, e^{p} & 19\end{array}$

6 Concluding remarks $\quad 21$

A On $\operatorname{sl}(2, \mathbb{R})$ Lie algebra $\quad 23$

A.1 $\mathrm{AdS}_{2}$ in global coordinates, another example 24

B Symmetries and conserved charges $\quad 25$

$\begin{array}{lll}\text { B.1 Ambiguities } & 26\end{array}$

$\begin{array}{ll}\text { B.2 } & \text { Iyer-Wald entropy }\end{array}$

$\begin{array}{ll}\text { C Computation of symplectic form } & 27\end{array}$

$\begin{array}{ll}\text { D Inner/outer horizons permutation symmetry } & 29\end{array}$

\section{Introduction}

Constructing and analyzing solutions to theories of (Einstein) gravity with various kind of matter fields in diverse dimensions has been a very active area of research since the conception of General Relativity. Black holes, stationary solutions with a regular event 
horizon, has been a class of solutions of particular interest. We now have classification (not necessarily a complete one) and in some case uniqueness theorems [1] for specific gravity theories. This classification is usually based on the choice of asymptotic behavior and horizon topology, the charges like mass, angular momenta and electric or magnetic (or possibly dipole) charges and, if there are "moduli" in the theory, on the asymptotic values of these moduli scalar fields. ${ }^{1}$

Based on the seminal works of Hawking [5] and Bekenstein [6], it was argued that black holes behave like thermodynamical systems and the four laws of black hole (thermo)dynamics was proposed [7]: black hole is a thermodynamical system at the Hawking temperature $T_{H}$ (the temperature of the Hawking radiation as seen by the asymptotic observer) and chemical potentials, the horizon angular velocities $\Omega^{i}$ and horizon electric/magnetic potentials $\Phi^{p}$. One can then associate conjugate charges to these, the angular momenta $J_{i}$, the electric/magentic charges $q_{p}$ and the (ADM) mass $M$. These parameters and charges satisfy first law of thermodynamics, if we associate an entropy $S_{B H}$ to the black hole, as Bekenstein and Hawking did; explicitly, ${ }^{2}$

$$
T_{H} \delta S_{B H}=\delta M-\sum_{i} \Omega^{i} \delta J_{i}-\sum_{a} \Phi^{p} \delta q_{p}
$$

The remarkable feature of thermodynamical description is its universality, that it is independent of the theory and the specific class of solutions in consideration; it stems from very deep connections between gravity and thermodynamics.

The next conceptual step in the thermodynamical description of black holes appeared in a series of papers by $\mathrm{R}$. Wald et al. $[9,10,12]$. It was argued that not only the charges $J_{i}, q_{p}$ and $M$, but also the entropy $S_{B H}$ may be viewed as a Noether conserved charge, associated with the Killing vector field which becomes null (and actually vanishes) at the horizon. Within this approach the first law of black hole thermodynamics was proved. Since our analysis will be based on $[9,10]$, we will review these works in appendix B. Among many novel features, Wald's approach clarified (1) how the charges $J_{i}, q_{p}, M$ and $S_{B H}$ depend on the theory (action), as well as the solution; (2) the significance of gravity equations of motion and dealing with "solutions" for having the thermodynamic description (recall that Noether charges are defined on-shell) and; (3) what is the meaning of "perturbations" $\delta X$ 's appearing in the first law (1.1): the first law is not only about some relations among the parameters defining the class of black hole solutions, the $\delta X$ 's are associated with the corresponding charges of a (non-stationary) system probing the black hole background specified by $T_{H}, \Omega^{i}$ and $\Phi^{p}$; the black hole is seen as a thermodynamical system by the probe.

In search for the micro/statistical mechanical system underlying black holes, the class of extremal black holes, those with $T_{H}=0$, proved very useful. Extremal black holes may be viewed as the ground state of a system with the same values of $J_{i}$ and $q_{p}$ and have

\footnotetext{
${ }^{1}$ This topic started off by notable papers of $\mathrm{W}$. Israel $[2,3]$, and is more than four decades old, with a rich literature, e.g. see $[1,4]$ and references therein as some examples.

${ }^{2}$ The moduli (the asymptotic value of scalar fields) may also appear in the first law through a modification of $\delta M$ term. Explicitly, through shifting $\delta M$ to $\delta M-\frac{\partial M}{\partial \phi_{\alpha}} \delta \phi_{\alpha}$ where $\phi_{\alpha}$ denotes the moduli [8].
} 
generically non-zero entropy, while at zero temperature. It was noted in [13-15] and then rigorously proved in a series of papers [16-18] that focusing on a region close to the horizon of extremal black holes we obtain a new class of solutions to the same theory of gravity. This class of solutions, the Near Horizon Extremal Geometries (NHEG's) have the same conserved charges, $J_{i}$ and $q_{p}$ as the original black hole, while have no horizon and have a different asymptotic region. As the near horizon limit has been taken, these geometries have no horizon and no singularity. The project of classification and uniqueness theorems for NHEG has been actively pursued in the last decade or so and we have several theorems in four and five dimensions (see [18] for a recent review). We will briefly review these in section 2 .

In this work we focus on the NHEG and construct three laws of NHEG (thermo)dynamics. We argue one may associate an entropy to the geometry as the Noether charge associated with a (class of) Killing vector field(s) which become null at specific points of spacetime, very similar to what Wald did for black holes. We then work out universal relations among the entropy and other Noether charges of the system. We also work out what resembles first law of (thermo)dynamics for black holes, i.e. a universal relation which governs the relation between perturbations in the entropy and other charges associated with the stationary or non-stationary perturbations of the NHEG.

The rest of this work is organized as follows. In section 2, we review some facts about the NHEG. In section 3, we compute all Noether charges associated with the symmetries of NHEG. In section 4, we present the three laws of NHEG mechanics. In section 4.1, we present zeroth law of NHEG mechanics. In section 4.2, work out the "entropy law" for the NHEG dynamics, i.e. a universal relation between entropy, which as we argue, itself is a Noether charge, and other Noether charges of the NHEG. The entropy law formula is closely related to Sen's entropy function [19]. In section 4.3, we construct "entropy perturbation law" for the NHEG. In section 5, we discuss whether the laws of NHEG dynamics can be constructed from those of black hole dynamics when the black hole becomes an extremal one. We end with discussions and concluding remarks. In the appendices we have gathered some useful relations about the $s l(2, \mathbb{R})$ algebra, a review of Wald-Iyer formulation of the entropy and the first law of black hole thermodynamics, details of the computation of the symplectic form used in section 4.3, and discuss the "inner-outer horizons permutation symmetry," used in section 5 .

\section{Near Horizon Extremal Geometries (NHEG)}

As mentioned in the introduction a generic black hole solution is determined by two class of parameters: those appearing in the thermodynamical description and those associated with the asymptotic values of moduli. There is a largely held idea that all thermodynamical black hole quantities is encoded only in the near horizon data. This viewpoint has been proved for the class of supersymmetric or BPS black holes where it has been shown that the value of the moduli fields at the horizon is independent of their asymptotic values and is completely determined by the (thermodynamical) conserved charges. This observation was called "attractor mechanism" [20-22]. It was then realized that $[14,15,19,23]$ ex- 
tremal black holes (which are not necessarily BPS) also exhibit attractor behavior. This means that all the information for "thermodynamical" description of black holes ${ }^{3}$ is already included in the NHEG. This prompted the study of extremal horizons and exploring the possibility of NHEG uniqueness theorems, which we will review in this section. For further details the reader is referred to the recent comprehensive review [18].

\subsection{Extremal horizons and near horizon limits}

Extremal black holes are solutions with vanishing surface gravity and hence they do not have a bifurcate horizon. Therefore, it is useful to describe them in a null Gaussian coordinate system [18]:

$$
d s^{2}=2 d \tilde{v}\left(d r+r \tilde{h}_{a}(r, x) d x^{a}+\frac{1}{2} r^{2} \tilde{F}(r, x) d \tilde{v}\right)+\tilde{\gamma}_{a b}(r, x) d x^{a} d x^{b},
$$

where the horizon is at $r=0$, and $\tilde{\gamma}_{a b}$ computed at $r=0$ is the metric on the horizon which is taken to be a smooth, non-degenerate, compact codimension two spacelike surface. One can then readily take the near horizon limit by expanding around $r=0$, setting $r=\epsilon \rho$ and $v=\tilde{v} / \epsilon, \epsilon \rightarrow 0$ to obtain

$$
d s^{2}=2 d v\left(d \rho+\rho h_{a}(x) d x^{a}+\frac{1}{2} \rho^{2} F(x) d v\right)+\gamma_{a b}(x) d x^{a} d x^{b},
$$

where $\gamma_{a b}(x)=\tilde{\gamma}_{a b}(0, x), h_{a}(x)=\tilde{h}_{a}(0, x), F(x)=\tilde{F}(0, x)$. The near-horizon limit has fixed all the $\rho$ dependence. Metric (2.2) has translation symmetry along $v$ coordinate, as well as scaling $(v, \rho) \rightarrow(v / \lambda, \lambda \rho)$.

Next, one should require (2.2) to also satisfy equations of motion. Depending on the theory and its matter content we have some different possibilities for the $h_{a}$ and $F$ functions and hence the symmetries of the $(v, \rho)$ space. In particular, for "static" cases with $d h_{a}=0$ and when the matter content satisfies strong energy condition the isometry of $(v, \rho)$ part enhances to $\mathrm{SL}(2, \mathbb{R})$. For stationary cases, with four and five dimensional Einstein-Maxwell-Dilaton (EMD) theory where metric on the space of $\mathrm{U}(1)$ gauge fields and dilatons is positive definite (they have non-negative kinetic term) and when the potential of the dilatons is non-positive again we are dealing with a background with $\mathrm{SL}(2, \mathbb{R}) \times \mathrm{U}(1)^{N}$ symmetry. Here we do not intend to review in detail the extremal horizon uniqueness theorems. For more detailed and precise discussion see [18].

As we see for physically interesting cases the symmetry of the extremal black hole geometry generically enhances to $\mathrm{SL}(2, \mathbb{R})$ and some other $\mathrm{U}(1)$ factors. Therefore, here we only focus on the geometries with such symmetry. Explicitly,

We define NHEG as the most general geometry with local $\mathrm{SL}(2, \mathbb{R}) \times \mathrm{U}(1)^{N}$ symmetry group.

Here, we consider a generic diffeomorphism and gauge invariant theory without specifying the explicit form of the action. (Note that EMD is a special class of such models.) In

\footnotetext{
${ }^{3}$ The term thermodynamical has been put in quotation because extremal black holes are systems at zero temperature and there is really no energy flow. This point will become more clear in the next sections.
} 
general, at most $d-3 \mathrm{U}(1)$ factors are associated with rotations of the $d$ dimensional spacetime while the rest of them (up to $N$ ) is the number of gauge fields.

For a generic NHEG we adopt a coordinate system which makes the $\mathrm{SL}(2, \mathbb{R}) \times \mathrm{U}(1)^{N}$ symmetry manifest:

$$
d s^{2}=\Gamma\left[-r^{2} d t^{2}+\frac{d r^{2}}{r^{2}}+\sum_{\alpha, \beta=1}^{d-n-2} \Theta_{\alpha \beta} d \theta^{\alpha} d \theta^{\beta}+\sum_{i, j=1}^{n} \gamma_{i j}\left(d \varphi^{i}+k^{i} r d t\right)\left(d \varphi^{j}+k^{j} r d t\right)\right]
$$

supplemented by a set of gauge fields $A^{(p)}$

$$
A^{(p)}=\sum_{i=1}^{n} f_{i}^{(p)}\left(d \varphi^{i}+k^{i} r d t\right)+e^{p} r d t
$$

In the above $i, j=1, \cdots, n$ and $p=n+1, \cdots, N$, and $n \leq d-3 . \Gamma, \Theta_{\alpha \beta}, \gamma_{i j}, f_{i}^{(p)}$ are functions of the polar coordinates $\theta^{\alpha}$ whose explicit form may be fixed upon imposing equations of motion. $k^{i}, e^{p}$ are constants, the constancy of which is a direct consequence of $\operatorname{SL}(2, \mathbb{R})$ symmetry. A full solution may also involve a number of scalars $\phi_{A}=\phi_{A}\left(\theta^{\alpha}\right)$, however, due to the attractor behavior (see [14] and references therein) the parametric dependence of the scalar fields is completely fixed by the other charges. So, while these scalars can affect the value of charges, we need not consider them separately in this paper. We take the constant $r, t$ surfaces, denoted by $H$, to be compact, smooth and non-degenerate. Moreover, we take the metric on $\varphi^{i}$ space, $\gamma_{i j}$, to be non-degenerate and positive definite.

The geometric part of the $\mathrm{SL}(2, \mathbb{R}) \times \mathrm{U}(1)^{N}$ symmetry, which is $\operatorname{SL}(2, \mathbb{R}) \times \mathrm{U}(1)^{n}$, is generated by the following Killing vector fields (cf. appendix A for our convention and notations for $\operatorname{sl}(2, \mathbb{R})$ algebra.)

$$
\begin{aligned}
\xi_{1} & =\partial_{t} \\
\xi_{2} & =t \partial_{t}-r \partial_{r} \\
\xi_{3} & =\frac{1}{2}\left(t^{2}+\frac{1}{r^{2}}\right) \partial_{t}-t r \partial_{r}-\sum_{i=1}^{n} \frac{k^{i}}{r} \partial_{\varphi^{i}} \\
m_{i} & =\partial_{\varphi^{i}}
\end{aligned}
$$

with the commutation relations:

$$
\begin{aligned}
{\left[\xi_{1}, \xi_{2}\right] } & =\xi_{1}, \quad\left[\xi_{2}, \xi_{3}\right]=\xi_{3}, \quad\left[\xi_{1}, \xi_{3}\right]=\xi_{2}, \\
{\left[\xi_{a}, m_{i}\right] } & =0, \quad a \in\{1,2,3\} \quad \text { and }, \quad i \in\{1, \ldots, n\} .
\end{aligned}
$$

\subsection{Relation between $\mathrm{SL}(2, \mathbb{R})$ and $\mathrm{U}(1)$ generators}

Let us define the $\operatorname{SL}(2, \mathbb{R})$ vector $n_{a}, a=1,2,3$ as the unit normal vector to $\operatorname{AdS}_{2}$ in the $\mathbb{R}^{2,1}$ embedding space, i.e. $n_{a} n^{a}=-1$. In the basis we have used for writing the metric (2.3) $n_{a}$ are (see appendix A for more discussions):

$$
n_{1}=-r, \quad n_{2}=-t r, \quad n_{3}=-\frac{t^{2} r^{2}-1}{2 r} .
$$


Using $n_{a}$, one has the following relation between the $\operatorname{SL}(2, \mathbb{R})$ isometries and $\mathrm{U}(1)$ symmetry generators:

$$
n^{a} \xi_{a}=k^{i} m_{i} .
$$

Note that we have used $\mathrm{SL}(2, \mathbb{R})$ metric (A.3) for raising $a$ index on $n_{a}$. To show this recall that the Killing vector $\xi_{3}$ is

$$
\xi_{3}=\frac{1}{2}\left(t^{2}+\frac{1}{r^{2}}\right) \partial_{t}-t r \partial_{r}-\sum_{i} \frac{k^{i}}{r} \partial_{\varphi^{i}}
$$

Multiplying by $r$ and rewriting the above equation in terms of Killing vectors yields:

$$
r \xi_{3}=-\frac{t^{2} r^{2}-1}{2 r} \xi_{1}+t r \xi_{2}-\sum_{i} k^{i} \partial_{\varphi^{i}}
$$

or

$$
n_{3} \xi_{1}-n_{2} \xi_{2}+n_{1} \xi_{3} \equiv n^{a} \xi_{a}=\sum_{i} k^{i} m_{i}
$$

More detailed analysis and useful identities about the $\operatorname{SL}(2, \mathbb{R})$ structure is gathered in the appendix A.

\section{NHEG conserved charges}

Given a geometry which is (a part of) a solution to a diffeomorphism invariant gravity theory, in the same spirit as the Noether theorem, one may associate a conserved quantity to each Killing vector field. A given solution may also be invariant under some "internal" symmetries, like in Maxwell theory, to which one may associate the corresponding Noether charges too. This general argument implies that with the NHEG with $\operatorname{SL}(2, \mathbb{R}) \times \mathrm{U}(1)^{N}$ symmetries one can associate $N+3$ conserved Noether charges. In this section we work out those charges. As reviewed in the appendix B, however, there are always ambiguities in defining Noether charge densities (specially when we are dealing with a symmetry associated with diffeomorphisms). These ambiguities are usually fixed by giving a reference point (e.g. asymptotic ADM charges). Here, we also discuss how those ambiguities may be dealt with in the NHEG case where we do not have a maximally symmetric asymptotic space. Here, following conventions of $[9,10]$, we use boldface for spacetime forms.

\subsection{Noether charge density of non-Abelian symmetries}

Obtaining Noether charge density $\mathbf{Q}$ from the Noether current $\mathbf{J}$ associated to a diffeomorphism generator (cf. appendix B) is not generally an easy task, but when we are dealing with non-Abelian symmetry groups, this will become straightforward due to construction we discuss below.

Consider a set of Killing vectors $\xi_{a}$ which satisfy the following Lie bracket relations

$$
\left[\xi_{a}, \xi_{b}\right]=f_{a b}^{c} \xi_{c}
$$


where $f_{a b}^{c}$ are the structural constants of the symmetry Lie algebra $\mathcal{G}$. Let $K_{a b}$ be the metric of the algebra. Then, noting that

$$
f_{a b}^{c} f^{a b d}=C_{2} K^{c d},
$$

where $C_{2}$ is the second rank Casimir of the algebra in the adjoint representation, we have

$$
\xi_{a}=\frac{1}{C_{2}} f_{a}^{b c}\left[\xi_{b}, \xi_{c}\right]
$$

(Note that the indices on the structure constant tensor is raised and lowered by metric $\left.K_{a b}.\right)$ Next, recalling the definition of the Lie bracket,

$$
\begin{aligned}
{\left[\xi_{b}, \xi_{c}\right]^{\mu} } & =\xi_{b}^{\nu} \nabla_{\nu} \xi_{c}^{\mu}-\xi_{c}^{\nu} \nabla_{\nu} \xi_{b}^{\mu} \\
& =\nabla_{\nu}\left(\xi_{b}^{[\nu} \xi_{c}^{\mu]}\right)
\end{aligned}
$$

In the second line we have used the Killing property $\nabla_{\nu} \xi^{\nu}=0$. Consequently, the Noether current $\mathbf{J}$ (introduced in (B.4)) may be written as

$$
\begin{aligned}
\mathbf{J}_{\xi_{a}}^{\mu} & =\boldsymbol{\Theta}_{\xi_{a}}^{\mu}-\mathcal{L} \xi_{a}^{\mu} \\
& =\frac{2}{C_{2}} \mathcal{L} f_{a}{ }^{b c} \nabla_{\nu}\left(\xi_{b}^{\mu} \xi_{c}^{\nu}\right)
\end{aligned}
$$

In the second line we have dropped $\boldsymbol{\Theta}_{\xi_{a}}$ term because it is a linear function of $\delta_{\xi_{a}} \Phi$ and for Killing fields $\delta_{\xi} \Phi=\mathcal{L}_{\xi} \Phi=0$. In our notations $\Phi$ stands for all the fields we have in our theory.

One can further simplify (3.5) using the chain rule and the fact that $\xi_{a}$ 's are isometries of $\mathcal{L}$, i.e. $\xi_{a}^{\nu} \nabla_{\nu} \mathcal{L}=0$, to obtain

$$
\mathbf{J}_{\xi_{a}}^{\mu}=\nabla_{\nu} \mathbf{Q}_{\xi_{a}}^{\mu \nu}
$$

in which

$$
\mathbf{Q}_{\xi_{a}}^{\mu \nu}=\frac{2}{C_{2}} \mathcal{L} f_{a}^{b c} \xi_{b}^{\mu} \xi_{c}^{\nu} .
$$

In the presence of (internal) gauge symmetries one should revisit the above analysis: in this case $\delta_{\xi} \Phi$ is not necessarily zero, $\delta_{\xi} \Phi$ should be zero up to internal gauge transformations, i.e. generically

$$
\delta_{\xi} \Phi=\delta_{\Lambda} \Phi, \quad \text { for some } \Lambda=\Lambda(\xi) .
$$

In the diffeomorphism and gauge invariant theories on which we have focused in this work, only the gauge fields $A_{\mu}^{(p)}$ are subject to the above discussion. So, let us revisit $\boldsymbol{\Theta}$ term for them:

$$
\begin{aligned}
\Theta^{\mu} & =\frac{\partial \mathcal{L}}{\partial \nabla_{\mu} A_{\nu}^{(p)}} \delta A_{\nu}^{(p)}=\frac{\partial \mathcal{L}}{\partial \nabla_{\mu} A_{\nu}^{(p)}} \partial_{\nu} \Lambda^{(p)} \\
& =\nabla_{\nu}\left(\frac{\partial \mathcal{L}}{\partial \nabla_{\mu} A_{\nu}^{(p)}} \Lambda^{(p)}\right)-\Lambda^{(p)} \nabla_{\nu} \frac{\partial \mathcal{L}}{\partial \nabla_{\mu} A_{\nu}^{(p)}},
\end{aligned}
$$

where $\Lambda^{(p)}=\Lambda^{(p)}\left(\xi_{a}\right)$ is determined such that $\delta_{\xi_{a}} A_{\mu}^{(p)}=\partial_{\mu} \Lambda^{(p)}$. 
Assuming that the action is local and invariant under the gauge $A \rightarrow A+d \Lambda$, it can only be a function of $F_{\mu \nu}=\partial_{[\mu} A_{\nu]}$ and the second term vanishes due to the field equations for gauge fields in the absence of source. ${ }^{4}$ Therefore,

$$
\Theta^{\mu}=\partial_{\nu} \mathbf{j}^{\mu \nu}, \quad \mathbf{j}^{\mu \nu}=\sum_{p} \Lambda^{(p)} \frac{\partial \mathcal{L}}{\partial F_{\mu \nu}^{(p)}} .
$$

This is the term that should be added to (3.7) in the presence of gauge fields and hence the complete form of the Noether charge density for the generator $\xi_{a}$ is ${ }^{5}$

$$
\mathbf{Q}_{\xi_{a}}^{\mu \nu}=\frac{2}{C_{2}} \mathcal{L} f_{a}^{b c} \xi_{b}^{\mu} \xi_{c}^{\nu}+\sum_{p} \Lambda^{(p)} \frac{\partial \mathcal{L}}{\partial F_{\mu \nu}^{(p)}} .
$$

\section{2 $\mathrm{SL}(2, \mathbb{R})$ conserved charges}

Applying the method of previous subsection, one can compute the conserved charges corresponding to $\mathrm{SL}(2, \mathbb{R})$ isometry of NHEG spacetime. It can be seen from (2.4) that

$$
\delta_{\xi_{1}} A^{(p)}=\delta_{\xi_{2}} A^{(p)}=0, \quad \delta_{\xi_{3}} A^{(p)}=-\frac{e^{p}}{r^{2}} d r=d\left(\frac{e^{p}}{r}\right)
$$

and hence $\Lambda_{\xi_{3}}^{(p)}=\frac{e^{p}}{r}$ (where $\Lambda_{\xi_{3}}^{(p)}$ is the one appearing in (3.9)).

For the $\operatorname{sl}(2, \mathbb{R})$ algebra, $C_{2}=2$ and the Noether charge density for generator $\xi_{a}$ becomes

$$
\mathbf{Q}_{a}^{\mu \nu}=\mathcal{L} f_{a}^{b c} \xi_{b}^{\mu} \xi_{c}^{\nu}+\delta_{a 3} \sum_{p} \frac{e^{p}}{r} \frac{\partial \mathcal{L}}{\partial F_{\mu \nu}^{(p)}} .
$$

Using this we can obtain conserved charges corresponding to $\operatorname{sl}(2, \mathbb{R})$ Killing vectors by integrating it over the closed surface $H$, which is any of $(d-2)$-dimensional $t, r=$ const surfaces in (2.3):

$$
\mathcal{Q}_{a} \equiv \oint_{H} d \Sigma_{\mu \nu} \mathbf{Q}_{a}^{\mu \nu}
$$

Replacing $\mathbf{Q}_{a}^{\mu \nu}$ from (3.14) and using (A.9) we obtain

$$
\mathcal{Q}_{a}=\frac{f_{a}^{b c}}{2} \delta_{\xi_{b}} n_{c} \oint_{H} d \Sigma_{t r} \mathcal{L}+\delta_{a 3} \sum_{p} \frac{e^{p}}{r} \oint_{H} d \Sigma_{\mu \nu} \frac{\partial \mathcal{L}}{\partial F_{\mu \nu}^{(p)}},
$$

where we have used the fact that any function of $r$ can be taken out of the integration, as the integration is on the constant $r$ surface $H$. Noting (A.8) and recalling the definition of the electric charge

$$
q_{p} \equiv-\oint_{H} \mathrm{~d} \Sigma_{\mu \nu} \frac{\partial \mathcal{L}}{\partial F_{\mu \nu}^{(p)}}
$$

\footnotetext{
${ }^{4}$ Note that $\mathrm{SL}(2, \mathbb{R})$ invariance does not allow for having local sources.

${ }^{5}$ This argument in a straightforward way extends to the non-Abelian internal gauge symmetries and also to the cases with higher dimensional $p$-forms. Moreover, it is possible that a black hole of non-trivial topology carries a dipole charge while it is neutral, e.g. as in the case of dipole black ring [24]. These dipole moments do appear in the first law [25] and our analysis may be extended to include these cases.
} 
we find

$$
\mathcal{Q}_{a}=n_{a} \oint_{H} d \Sigma_{t r} \mathcal{L}-\delta_{a 3} \sum_{p} \frac{e^{p}}{r} q_{p}
$$

It will be more useful to consider the $\mathrm{SL}(2, \mathbb{R})$ invariant linear combinations of charges $\mathcal{Q}_{a}$ by multiplying both sides with $n^{a}$, to obtain

$$
n^{a} \mathcal{Q}_{a}=\sum_{p} e^{p} q_{p}-\oint_{H} d \Sigma_{t r} \mathcal{L} .
$$

The above analysis, which is based on Noether's theorem, makes it apparent that despite explicit $t, r$ dependence, $\mathcal{Q}_{a}$ 's are conserved. Moreover, in writing $\operatorname{SL}(2, \mathbb{R})$ charges (3.18) we have already fixed the ambiguities associated with Noether-Wald charges discussed in appendix B. This point will be discussed further in section 4.2.

\subsection{NHEG entropy as a conserved charge}

Despite the fact that the NHEG does not have a (Killing) horizon as black holes do, recalling that they can be obtained as the near horizon limit of extremal black holes, one may formally associate an entropy to them. To this end, we note that instead of the horizon, the NHEG have surfaces $H$ (i.e. surfaces of constant time and radius in the coordinates used to represent the NHEG metric (2.3)). As discussed in the appendix A, SL $(2, \mathbb{R})$ invariance facilitates defining an $(\mathrm{SL}(2, \mathbb{R})$ invariant) binormal 2 -form (which is dual to the volume form on $H$ ). Given these, we can readily write the analogue of Iyer-Wald entropy [10] for the NHEG:

Definition. Entropy of the NHEG as a solution of the e.o.m is defined as

$$
\begin{aligned}
\frac{S}{2 \pi} & \equiv-\oint_{H} \operatorname{Vol}(H) \frac{\delta \mathcal{L}}{\delta R_{\mu \nu \alpha \beta}} \epsilon_{\mu \nu} \epsilon_{\alpha \beta} \\
& =-2 \oint_{H} \mathrm{~d} \Sigma_{\mu \nu} E^{\mu \nu \alpha \beta} \epsilon_{\alpha \beta},
\end{aligned}
$$

where $H$ is any of the $\mathrm{SL}(2, \mathbb{R})$ invariant $(d-2)$-dimensional surfaces, $\epsilon_{\mu \nu}$ is the $\operatorname{SL}(2, \mathbb{R})$ invariant binormal 2-form, cf. (A.10), and $E^{\mu \nu \alpha \beta} \equiv \frac{\delta \mathcal{L}}{\delta R_{\mu \nu \alpha \beta}}$.

One of the key steps in Wald formulation of "entropy as a Noether charge" [9] is the realization that Killing horizon is associated with a null Killing vector whose dual oneform vanishes on the horizon. In the NHEG we do not have the Killing horizon, however, recalling discussions in section 2.2, we indeed have an infinite family of such Killing vector fields:

$$
\zeta_{H} \equiv n_{H}^{a} \xi_{a}-k^{i} m_{i}
$$

where $n_{H}^{a}=n^{a}\left(t=t_{H}, r=r_{H}\right)$ and $n_{a}$ is given in (2.9). We will prove the following proposition:

Conserved charge corresponding to Killing vector $\zeta_{H}$ is the NHEG Entropy, defined in (3.20). 
Proof. We first note that $\zeta_{H}$ is a linear combination of Killing vector fields with constant coefficients ( $n_{H}^{a}$ and $k^{i}$ are constants), and hence $\zeta_{H}$ is a Killing vector field. Next, we note that according to the proposition 4.1 of the Iyer-Wald paper [10] (see appendix B), the Noether conserved charge corresponding to $\zeta_{H}$ can be decomposed as

$$
\mathcal{Q}_{\zeta_{H}}=\oint_{H} d \Sigma_{\mu \nu}\left(W^{\mu \nu}{ }_{\alpha} \zeta_{H}^{\alpha}-2 \mathbf{E}^{\mu \nu}{ }_{\alpha \beta} \nabla^{\alpha} \zeta_{H}^{\beta}+Y^{\mu \nu}+(d Z)^{\mu \nu}\right),
$$

where $\mathbf{E}^{\mu \nu \alpha \beta}=\frac{\delta \mathcal{L}}{\delta R_{\mu \nu \alpha \beta}}$ and $W$ and $Y$ and $Z$ are covariant quantities which are locally constructed from fields and their derivatives. $Y$ is linear in $\delta_{\zeta_{H}} \Phi$ and $Z$ is linear in $\zeta_{H}$ (recall (2.9) and (2.10)). As discussed in the previous section, $\delta_{\zeta_{H}} \Phi=0$ up to internal gauge transformations. In our case, that is, all $\delta_{\xi} \Phi=0$, except for $\delta_{\xi_{3}} A^{(p)}$ which is a pure gauge. We fix the $Y$ ambiguity requiring physical charges to be gauge independent. The $W$ and $d Z$ ambiguities are removed, noting that the Killing vector field $\zeta_{H}$ has been constructed such that $\left.\zeta_{H}\right|_{t=t_{H}, r=r_{H}}=0$. Therefore,

$$
\mathcal{Q}_{\zeta_{H}}=-2 \oint_{H} d \Sigma_{\mu \nu} \mathbf{E}^{\mu \nu}{ }_{\alpha \beta} \nabla^{\alpha} \zeta_{H}^{\beta}
$$

To determine $\nabla^{\alpha} \zeta_{H}^{\beta}$, we take covariant derivative of both sides of the identity (2.10),

$$
n^{a} \nabla^{\alpha} \xi_{a}^{\beta}-k^{i} \nabla^{\alpha} m_{i}^{\beta}=-\xi_{a}^{\beta} \nabla^{\alpha} n^{a}=\epsilon^{\alpha \beta}
$$

where in the second equation we have (A.10). The l.h.s. of the above equality may be computed at any $r, t$. In particular, when computed at $r=r_{H}, t=t_{H}$ we obtain

$$
\nabla^{\alpha} \zeta_{H}^{\beta}=\epsilon^{\alpha \beta}
$$

With the above (3.23) takes the form

$$
\mathcal{Q}_{\zeta_{H}}=-2 \oint_{H} d \Sigma_{\mu \nu} \mathbf{E}^{\mu \nu}{ }_{\alpha \beta} \epsilon^{\alpha \beta}=\frac{S}{2 \pi},
$$

which is exactly the NHEG entropy (3.20) calculated on the surface $H$. It is important to note that although the surface $H$ (defined at constant $t_{H}, r_{H}$ ) has appeared in the above arguments, the final result is independent of $t_{H}$ and $r_{H}$. In other words, there are infinitely many Killing vector fields $\zeta_{H}$, all leading to the same entropy. This is of course expected because of the $\operatorname{SL}(2, \mathbb{R})$ invariance.

\section{Laws of NHEG dynamics}

In this section we derive three laws of NHEG mechanics. The first two are describing the NHEG geometry itself, but the third one governs perturbations (or probes) over the NHEG background. The first and third laws resemble the laws of black hole mechanics [7], while "entropy law" has no counterpart for generic black holes. 


\subsection{Zeroth law of NHEG dynamics}

Demanding $(2.3)$ to be $\mathrm{SL}(2, \mathbb{R})$ invariant, restricts $k^{i}$ and $e^{p}$ parameters, while imposing equations of motion will determine other functions there. In particular, $\xi_{3}$ is a Killing vector field only if $\nabla^{\theta^{\alpha}} \xi_{3}^{\varphi^{i}}+\nabla^{\varphi^{i}} \xi_{3}^{\theta^{\alpha}} \sim \partial_{\theta^{\alpha}} k^{i}=0$. Similarly, if we require that $\mathcal{L}_{\xi_{3}} F^{(p)}=0$, where $F^{(p)}=d A^{(p)}$ and $\mathcal{L}_{\xi_{3}}$ denotes the Lie derivative w.r.t. the Killing vector $\xi_{3}$, leads to $\partial_{\theta^{\alpha}} e^{p}=0$. That is, $k^{i}$ 's and $e^{p}$ 's should be constants with respect to the coordinates $\theta^{\alpha}$.

The constancy of $k^{i}$ and $e^{p}$ can be treated as the zeroth law of NHEG dynamics.

In section 5, we discuss the relation between the NHEG and (near) extremal black holes and show the close connection between the NHEG zeroth law and the constancy of Hawking temperature and horizon angular velocities. This makes the analogy of NHEG zeroth law and the black hole zeroth law.

\subsection{NHEG entropy law}

In this section we prove the "NHEG entropy law":

$$
\frac{S}{2 \pi}=k^{i} J_{i}+e^{p} q_{p}-\oint_{H} \sqrt{-g} \mathcal{L},
$$

where $k^{i}$ and $e^{p}$ are constants appearing in the NHEG solution (2.3) and (2.4), $J_{i}$ and $q_{p}$ denote the corresponding $N \mathrm{U}(1)$ charges and $\sqrt{-g}=\Gamma^{d / 2} \sqrt{\operatorname{det} \Theta_{\alpha \beta} \cdot \operatorname{det} \gamma_{i j}}$.

Derivation: We start by taking covariant derivative from (3.21)

$$
-\nabla^{\alpha} \zeta_{H}^{\beta}=k^{i} \nabla^{\alpha} m_{i}^{\beta}-n_{H}^{a} \nabla^{\alpha} \xi_{a}^{\beta},
$$

and integrating both sides over $2 \oint_{H} d \Sigma_{\mu \nu} \mathbf{E}_{\alpha \beta}^{\mu \nu}$ :

$$
-2 \oint_{H} d \Sigma_{\mu \nu} \mathbf{E}_{\alpha \beta}^{\mu \nu} \nabla^{\alpha} \zeta_{H}^{\beta}=2 \oint_{H} d \Sigma_{\mu \nu} \mathbf{E}_{\alpha \beta}^{\mu \nu}\left(k^{i} \nabla^{\alpha} m_{i}^{\beta}-n_{H}^{a} \nabla^{\alpha} \xi_{a}^{\beta}\right) .
$$

Next, we note that as discussed in the appendix B, there is a Noether conserved charge associated each of the Killing vector fields $\zeta_{H}, \xi_{a}$ and $m_{i}$, but these conserved charges come with three kind of $W, Y, d Z$ ambiguities

$$
\begin{aligned}
\mathcal{Q}_{\zeta_{H}} & =\oint_{H} d \Sigma_{\mu \nu}\left[W_{\alpha}^{\mu \nu} \zeta_{H}^{\alpha}-2 \mathbf{E}_{\alpha \beta}^{\mu \nu} \nabla^{\alpha} \zeta_{H}{ }^{\beta}+Y_{\zeta_{H}}^{\mu \nu}+\left(d Z_{\zeta_{H}}\right)^{\mu \nu}\right], \\
\mathcal{Q}_{m_{i}} & =\oint_{H} d \Sigma_{\mu \nu}\left[W_{\alpha}^{\mu \nu} m_{i}^{\alpha}-2 \mathbf{E}^{\mu \nu}{ }_{\alpha \beta} \nabla^{\alpha} m_{i}^{\beta}+Y_{m_{i}}^{\mu \nu}+\left(d Z_{m_{i}}\right)^{\mu \nu}\right], \\
\mathcal{Q}_{\xi_{a}} & =\oint_{H} d \Sigma_{\mu \nu}\left[W_{\alpha}^{\mu \nu} \xi_{a}^{\alpha}-2 \mathbf{E}^{\mu \nu}{ }_{\alpha \beta} \nabla^{\alpha} \xi_{a}^{\beta}+Y_{\xi_{a}}^{\mu \nu}+\left(d Z_{\xi_{a}}\right)^{\mu \nu}\right] .
\end{aligned}
$$

Computed "at the horizon" where $\zeta_{H}$ is zero, the $W$ and $d Z$ terms in $\mathcal{Q}_{\zeta_{H}}$ vanish. Similarly, in the following linear combination of other charges

$$
\sum_{a} n_{H}^{a} \mathcal{Q}_{\xi_{a}}-\sum_{i} k^{i} \mathcal{Q}_{m_{i}}
$$


the $W$ and $d Z$ terms also vanish. Therefore, (4.3) becomes

$$
\mathcal{Q}_{\zeta_{H}}-\left(\sum_{a} n_{H}^{a} \mathcal{Q}_{\xi_{a}}-\sum_{i} k^{i} \mathcal{Q}_{m_{i}}\right)=\oint_{H} d \Sigma_{\mu \nu}\left(Y_{\zeta_{H}}^{\mu \nu}-n_{H}^{a} Y_{\xi_{a}}^{\mu \nu}+k^{i} Y_{m_{i}}^{\mu \nu}\right)
$$

The r.h.s. of the above equation is zero because $\delta_{\xi} \Phi$ is linear in $\xi$ (or in $\nabla \xi$ ) as well as in $\Phi$ (or in $\nabla \Phi)$, and hence $\delta_{\zeta_{H}} \Phi-\left(n_{H}^{a} \delta_{\xi_{a}} \Phi-k^{i} \delta_{m_{i}} \Phi\right)=\delta_{\zeta_{H}-n_{H}^{a} \xi_{a}+k^{i} m_{i}} \Phi=0$. In summary, all the three $W, Y$ and $d Z$ type ambiguities cancel out from the two sides of the equality and we obtain

$$
\mathcal{Q}_{\zeta_{H}}=\sum_{a} n_{H}^{a} \mathcal{Q}_{\xi_{a}}-\sum_{i} k^{i} \mathcal{Q}_{m_{i}}
$$

With a similar reasoning one can show that the above equation holds when we replace $\mathcal{Q}_{\zeta_{H}}$ by $S /(2 \pi)$ (cf. (3.26)), $\mathcal{Q}_{m_{i}}$ by physical angular momenta $J_{i}$, and $n_{H}^{a} \mathcal{Q}_{\xi_{a}}$ from (3.19). We hence obtain the desired entropy law expression (4.1).

Before closing this section some comments are in order:

1. eq.(4.1) is universal, meaning that it is the relation between conserved charges associated with any NHEG solution to any diffeomorphism invariant theory (of gravity).

2. In the above we have used the fact that the l.h.s. of (3.19) is $\operatorname{SL}(2, \mathbb{R})$ invariant and hence can be computed at any arbitrary constant $t, r$ surface.

3. The entropy law (4.1) is a manifestation of the fact that the $\operatorname{SL}(2, \mathbb{R})$ and $\mathrm{U}(1)$ generators mix with each other, as is manifest, e.g. from (2.5). Explicitly, the $\xi_{3}$ Killing vector also involves a $k^{i} \partial_{\phi^{i}}$ term (2.5).

4. The entropy law (and also the entropy perturbation law (4.5)) are invariant under permutation of $N \mathrm{U}(1)$ symmetries.

5. We stress that such a universal relation between entropy and other thermodynamical quantities/conserved charges does not exist for generic black holes. As we will discuss further in following sections, the "first law" of black hole thermodynamics deals with perturbations of these parameters and not themselves. Note also that Smarr-like formulas which may resemble our entropy law, are not universal and are solution and/or theory dependent.

6. The reason why our derivation of entropy law (or in other words, Wald's derivation) does not hold for generic black holes is presence of ambiguities we discussed in some detail, and in particular the fact that these ambiguities should be computed and compared at different locations in the black hole geometry. In our case, unlike the black hole case, we have vanishing Killing vector $\zeta_{H}$ for any $t_{H}, r_{H}$. We will elaborate on this point further in the next sections.

7. Our derivation is based on Noether conserved charges and hence makes clear the role of being on-shell. In particular, in the last term in (4.1), the Lagrangian $\mathcal{L}$ should be computed on the NHEG solution. 
8. The entropy (3.26) is a conserved charge associated with a vanishing Killing vector field $\zeta_{H}$, although NHEG does not have a horizon. The entropy is completely determined by the geometry and not other fields, although other fields affect the geometry through Einstein equations.

9. Note that in our ansatz for gauge fields (2.4) we have already included possibility of having a non-zero magnetic flux (through the $f_{i}^{(p)} d \varphi^{i}$ term). As expected, the magnetic and electric flux (denoted through $e^{p}$ ) appear asymmetrically in our entropy law; magnetic flux appears only through the Lagrangian term.

10. In our derivation it is clear that the terms in the r.h.s. of the entropy law are associated with $N \mathrm{U}(1)$ symmetries of the system and the corresponding conserved charges. The dilaton-type scalar fields (or moduli) which are not associated with any symmetry can only appear through the Lagrangian term. This is a realization of the attractor behavior $[14,15,23]$ in our setup.

11. Our entropy law is closely related to Sen's entropy function $[14,19] .{ }^{6}$ However, our derivation is quite different; specifically we note that our derivation is completely based on the NHEG and not the extremal black hole. Therefore, we need not deal with the issues which may arise in the usage of Wald entropy formula which is derived for bifurcate horizons, for extremal horizons. Further discussion related to this point can be found in section 5 .

\subsection{NHEG entropy perturbation law}

In the previous section we derived the NHEG entropy law, which is a relation among conserved Noether-Wald charges of the NHEG which is a solution to equations of motion for a given gravity theory with our desired $\mathrm{SL}(2, \mathbb{R}) \times \mathrm{U}(1)^{N}$ symmetry. As pointed out this relation has no universal analog for generic black holes. In this section we construct the analog of the first law of thermodynamics for the NHEG.

To this end, let us denote the NHEG solution by the field configuration $\Phi_{0}$ and consider a perturbation around it $\delta \Phi$. The configuration $\Phi_{0}+\delta \Phi$ is not necessarily of the form of NHEG, however, we assume that the perturbations $\delta \Phi$ satisfy linearized equations of motion around the NHEG background solution $\Phi_{0}$. Therefore, $\delta \Phi$ can also be labeled by the same charges as the background. Let us denote these charges by $\delta J_{i}, \delta q_{p}$ and $\delta S$. Our discussions here are basically paralleling those in [10] for ordinary black hole. However, as we will see below, the case of NHEG has its own specific and novel features. Under specific conditions over field perturbations $\delta \Phi$ which are listed in the end of this section, we prove the "entropy perturbation law" relating different charges of the probe:

$$
\frac{\delta S}{2 \pi}=k^{i} \delta J_{i}+e^{p} \delta q_{p}
$$

\footnotetext{
${ }^{6}$ We point out that in the entropy function formulation one is prescribed to start from an "off-shell entropy functional" defined on the NHEG solution (2.3) and (2.4), and then find equations of motion by setting zero variations of this entropy functional with respect to unknown functions or parameters of the NHEG solution ansatz. Computing the value of this entropy functional on the solutions to these equations of motion is shown to reproduce Wald entropy for extremal black holes [14, 23].
} 
Derivation: Noether current corresponding to the diffeomorphism generated by $\zeta_{H}$ is (see appendix B for notations):

$$
\mathbf{J}_{\zeta_{H}}=\boldsymbol{\Theta}\left(\Phi, \delta_{\zeta_{H}} \Phi\right)-\zeta_{H} \cdot \mathbf{L}
$$

where $\zeta_{H}$ is the Killing vector field defined in (3.21). We will use $\xi \cdot \mathbf{X}$ to denote the contraction of the vector $\xi$ with the first index of the form $\mathbf{X}$, which is usually written as $i_{\xi} \mathbf{X}$. Let us now consider variations in (4.6) associated with $\Phi_{0} \rightarrow \Phi_{0}+\delta \Phi$ :

$$
\delta \mathbf{J}_{\zeta_{H}}=\delta\left[\Theta\left(\Phi, \delta_{\zeta_{H}} \Phi\right)\right]-\zeta_{H} \cdot \delta \mathbf{L} .
$$

We assume that the variations do not alter the quantities attributed to the background. In particular, this means that $\delta \zeta_{H}, \delta \xi_{a}, \delta m_{i}$ are all vanishing (as they do in the case of black holes $[9,10])$. In this sense these variations are considered as perturbations or probes over the NHEG. Let us start our analysis from the last term in (4.7):

$$
\delta \mathbf{L}=\mathbf{E}_{i} \delta \Phi^{i}+\mathrm{d} \mathbf{\Theta}\left(\Phi_{0}, \delta \Phi\right) .
$$

The first term vanishes due to the on-shell condition and the second term is simplified recalling the identity $\xi \cdot \mathrm{d} \boldsymbol{\Theta}=\delta_{\xi} \boldsymbol{\Theta}-\mathrm{d}(\xi \cdot \boldsymbol{\Theta})$ which is valid for any diffeomorphism $\xi$, therefore,

$$
\zeta_{H} \cdot \delta \mathbf{L}=\delta_{\zeta_{H}} \Theta\left(\Phi_{0}, \delta \Phi\right)-\mathrm{d}\left(\zeta_{H} \cdot \Theta\left(\Phi_{0}, \delta \Phi\right)\right) .
$$

Inserting the above into (4.7) we obtain

$$
\delta \mathbf{J}_{\zeta_{H}}=\boldsymbol{\omega}\left(\Phi_{0}, \delta \Phi, \delta_{\zeta_{H}} \Phi\right)+\mathrm{d}\left(\zeta_{H} \cdot \boldsymbol{\Theta}\left(\Phi_{0}, \delta \Phi\right)\right) .
$$

where

$$
\boldsymbol{\omega}\left(\Phi_{0}, \delta_{1} \Phi, \delta_{2} \Phi\right) \equiv \delta_{1} \boldsymbol{\Theta}\left(\Phi_{0}, \delta_{2} \Phi\right)-\delta_{2} \boldsymbol{\Theta}\left(\Phi_{0}, \delta_{1} \Phi\right)
$$

is the symplectic current, the $(d-1)$-form associated with variations $\delta_{1}, \delta_{2}$, and is bilinear in its arguments [9]. This implies that for Killing vectors $\xi$ with $\delta_{\xi} \Phi_{0}=0$, the symplectic form vanishes. However, in presence of gauge fields $\delta_{\xi} \Phi_{0}$ need not vanish for a symmetry, it may be non-zero up to gauge transformations. In particular, as we have already seen in previous section, this is the case for the third Killing vector $\xi_{3}$ and the corresponding symplectic current $\boldsymbol{\omega}\left(\Phi_{0}, \delta \Phi, \delta_{\xi_{3}} \Phi\right)$ does not vanish. This feature (which was not relevant for the discussions of black holes $[9,10]$ ) has an important role in our derivation of the entropy perturbation law.

The current $\mathbf{J}_{\zeta_{H}}$ is conserved on-shell, i.e. $\mathrm{d} \mathbf{J}_{\zeta_{H}}=0$, so one can associate a conserved charge $d-2$ form $\mathbf{Q}_{\zeta_{H}}, \mathbf{J}_{\zeta_{H}}=\mathrm{d} \mathbf{Q}_{\zeta_{H}}$, to the symmetry generated by $\zeta_{H}$. Moreover, when the solution is deformed by a perturbation which is a solution to the linearized equations of motion, the relation $\mathrm{d} \mathbf{J}_{\zeta_{H}}=0$ still holds even if the perturbation is not symmetric under $\zeta_{H}$ (i.e. $\delta_{\zeta_{H}}(\delta \Phi) \neq 0$ ). In other words, one can take the variation of the relation $\mathbf{J}_{\zeta_{H}}=\mathrm{d} \mathbf{Q}_{\zeta_{H}}$ and arrive at $[9]$

$$
\delta \mathbf{J}_{\zeta_{H}}=\delta \mathrm{d} \mathbf{Q}_{\zeta_{H}}=\mathrm{d} \delta \mathbf{Q}_{\zeta_{H}}
$$


From the above equation, we also learn that perturbations over a background can be labeled by the charges corresponding to the background symmetries, although they do not carry those symmetries. Using (4.12) in (4.10) yields

$$
\boldsymbol{\omega}\left(\Phi_{0}, \delta \Phi, \delta_{\zeta_{H}} \Phi\right)=\mathrm{d}\left(\delta \mathbf{Q}_{\zeta_{H}}-\zeta_{H} \cdot \boldsymbol{\Theta}\left(\Phi_{0}, \delta \Phi\right)\right)
$$

We integrate the above "conservation equation" over a timelike hypersurface $\Sigma$ bounded between two radii $r=r_{H}, r=\infty$. The hypersurface $\Sigma$ can be simply chosen as a constant time surface $t=t_{H}$. The interior boundary $r=r_{H}$ is necessary, since $\mathrm{AdS}_{2}$ does not have a compact interior. As discussed before, the surface $H$ will play the role of horizon on which we define the entropy of NHEG. The $r=\infty$ choice for the other boundary, is a convenient choice because the extra terms appearing due to gauge transformations vanish (cf. appendix C, and in particular discussions around (C.10)). Following [9], we define the symplectic form associated with $\Sigma$ as

$$
\Omega\left(\Phi_{0}, \delta_{1} \Phi, \delta_{2} \Phi\right) \equiv \int_{\Sigma} \boldsymbol{\omega}\left(\Phi_{0}, \delta_{1} \Phi, \delta_{2} \Phi\right)
$$

Integrating (4.13) over $\Sigma$ then yields:

$$
\begin{aligned}
\Omega\left(\Phi_{0}, \delta \Phi, \delta_{\zeta_{H}} \Phi\right) & =\oint_{\partial \Sigma}\left(\delta \mathbf{Q}_{\zeta_{H}}-\zeta_{H} \cdot \boldsymbol{\Theta}\left(\Phi_{0}, \delta \Phi\right)\right) \\
& =\oint_{\infty}\left(\delta \mathbf{Q}_{\zeta_{H}}-\zeta_{H} \cdot \boldsymbol{\Theta}\left(\Phi_{0}, \delta \Phi\right)\right)-\oint_{H} \delta \mathbf{Q}_{\zeta_{H}}
\end{aligned}
$$

where in the first line we have used the Stokes theorem to convert the integral over $\Sigma$ to an integral over its boundary $\partial \Sigma$ and in the second line, we used the fact that $\zeta_{H}=n_{H}^{a} \xi_{a}-k^{i} m_{i}$ vanishes on $H$. Since the charge perturbation $\delta \mathbf{Q}_{\zeta_{H}}$ is linear in the vector $\zeta_{H}$, one can expand the first term on r.h.s. of (4.15)

$$
\Omega\left(\Phi_{0}, \delta \Phi, \delta_{\zeta_{H}} \Phi\right)=n_{H}^{a} \oint_{\infty}\left(\delta \mathbf{Q}_{a}-\xi_{a} \cdot \boldsymbol{\Theta}\right)-k^{i} \oint_{\infty}\left(\delta \mathbf{Q}_{m_{i}}-m_{i} \cdot \boldsymbol{\Theta}\right)-\oint_{H} \delta \mathbf{Q}_{\zeta_{H}}
$$

$m_{i}$ is tangent to the boundary surface and hence the pullback of $m_{i} \cdot \boldsymbol{\Theta}$ over the surface $r=\infty$ vanishes, and we have

$$
\Omega\left(\Phi_{0}, \delta \Phi, \delta_{\zeta_{H}} \Phi\right)=n_{H}^{a} \delta \mathcal{E}_{a}-k^{i} \oint_{\infty} \delta \mathbf{Q}_{m_{i}}-\oint_{H} \delta \mathbf{Q}_{\zeta_{H}}
$$

where

$$
\delta \mathcal{E}_{a} \equiv \oint_{\infty}\left(\delta \mathbf{Q}_{\xi_{a}}-\xi_{a} \cdot \boldsymbol{\Theta}\right)
$$

is the canonical generator of the symmetry $\xi_{a}$ in the covariant phase space [11].

The technical details of computation of $\Omega\left(\Phi_{0}, \delta \Phi, \delta_{\zeta_{H}} \Phi\right)$ is given in the appendix C, where it is shown that

$$
\Omega\left(\Phi_{0}, \delta \Phi, \delta_{\zeta_{H}} \Phi\right)=-e^{p} \delta q_{p}
$$


Substituting this result into (4.17) yields

$$
\oint_{H} \delta \mathbf{Q}_{\zeta_{H}}=k^{i} \delta J_{i}+e^{p} \delta q_{p}+n_{H}^{a} \delta \mathcal{E}_{a},
$$

where $\delta J_{i}$ is the angular momentum corresponding to the rotational symmetry $m_{i}$

$$
\delta J_{i} \equiv-\oint_{\infty} \delta \mathbf{Q}_{m_{i}} .
$$

(Since pullback of $m_{i} \cdot \boldsymbol{\Theta}$ vanishes over any constant $t, r$ surface on NHEG, one can show that in the above equation $\delta J_{i}$ could be computed with the integral at $\infty$ replaced by any $r=r_{H}$ surface.)

To show that the left side of (4.19) is actually the perturbation of entropy $\delta S$, we should discuss ambiguities of $\delta \mathbf{Q}_{\zeta_{H}}$. Any Noether charge can be decomposed as in (3.22) with $W, Y$ and $d Z$ ambiguities. The $W$ and $d Z$ ambiguities vanish since they are linear in $\zeta_{H}$, which vanishes at surface $H$. The $\delta Y$ ambiguity, which is proportional to variation of fields $\delta_{\xi} \Phi$ needs more attention. Since $\zeta_{H}=0$, at surface $H, \delta_{\zeta_{H}} \Phi=0$. This implies that $\mathrm{Y}$ vanishes on background over $H$, and also that its perturbation is given by

$$
\begin{aligned}
\delta Y\left(\Phi_{0}, \delta_{\zeta_{H}} \Phi\right) & =Y\left(\Phi_{0}, \delta \delta_{\zeta_{H}} \Phi\right) \\
& =Y\left(\Phi_{0}, \delta_{\zeta_{H}} \delta \Phi\right) \\
& =\delta_{\zeta_{H}} Y\left(\Phi_{0}, \delta \Phi\right) \\
& =\zeta_{H} \cdot d Y+d\left(Y \cdot \zeta_{H}\right) .
\end{aligned}
$$

In the above we have used the fact that since $\delta \zeta_{H}=0$, we can interchange $\delta_{\zeta_{H}}$ and $\delta$. Equation (4.21) is linear in the generator $\zeta_{H}$, does not contribute to the left hand side of (4.19) and therefore

$$
\delta \oint_{H} \mathbf{Q}_{\zeta_{H}}=-2 \delta \oint_{H} d \Sigma_{\mu \nu} \mathbf{E}_{\alpha \beta}^{\mu \nu} \nabla^{\alpha} \zeta_{H}^{\beta}=\frac{\delta S}{2 \pi}
$$

so

$$
\frac{\delta S}{2 \pi}=k^{i} \delta J_{i}+e^{p} \delta q_{p}+n_{H}^{a} \delta \mathcal{E}_{a} .
$$

Analysis of $[26,27]$ indicates that the NHEG background is stable for a class of field perturbation which satisfy certain boundary conditions. As we will show in our upcoming work [28], this stability condition implies $\delta \mathcal{E}_{a}=0$. Dropping the last term in (4.23) by the choice of boundary conditions, we arrive at the desired entropy perturbation law (4.5).

To end this section we summarize the assumptions over the field perturbations which resulted in the entropy perturbation law (4.5):

- Perturbations should satisfy the linearized field equations.

- Perturbations are restricted to those for which $\operatorname{SL}(2, \mathbb{R})$ charges vanish, i.e. $\delta \mathcal{E}_{a}=0$. This is typically done by choosing a set of boundary conditions.

We also note that the variation $\delta$ does not affect the Killing vectors associated with the background, i.e. $\delta \zeta_{H}=\delta \xi_{a}=\delta m_{i}=0$. 


\section{$5 \quad$ NHEG vs. extremal black hole}

So far we focused on NHEG as an interesting class of solutions to gravity theories and introduced and worked out three laws of NHEG dynamics. NHEG, as the name implies, is related to extremal black holes and one may wonder if laws of NHEG dynamics can be (directly) related to the laws of extremal black hole thermodynamics. This question has of course been discussed and studied in the literature from various different perspectives, see in particular $[29,30]$. This section is mainly meant to fill some gaps remaining in the literature about the connection of NHEG and extremal black holes.

The most general form of the metric of a stationary and axisymmetric black hole possessing some $\mathrm{U}(1)$ gauge fields, can be written in the ADM form as

$$
\begin{aligned}
d s^{2} & =-f d \tau^{2}+g_{\rho \rho} d \rho^{2}+\tilde{g}_{\alpha \beta} d \theta^{\alpha} d \theta^{\beta}+g_{i j}\left(d \psi^{i}-\omega^{i} d \tau\right)\left(d \psi^{j}-\omega^{j} d \tau\right), \\
\tilde{A}^{(p)} & =\Phi^{(p)} d \tau+\sum_{i} \mu_{i}^{(p)}\left(d \psi^{i}-\omega^{i} d \tau\right),
\end{aligned}
$$

where $f, g_{\rho \rho}, \tilde{g}_{\alpha \beta}, g_{i j}, \omega^{i}$ and $\Phi^{(p)}, \mu_{i}^{(p)}$ are functions of $\rho, \theta^{\alpha}$ and $i, j=1,2, \cdots, n$ and $p=$ $n+1, \cdots, N$. The horizons of black hole are at the roots of $g^{\rho \rho}$,

$$
g_{\rho \rho}=\frac{1}{D^{2}\left(\rho, \theta^{\alpha}\right) \Delta(\rho)}, \quad \Delta=\prod_{m}\left(\rho-r_{m}\right),
$$

where we assume the function $D$ to be analytic and nonvanishing everywhere. Due to the smoothness of metric on the horizons $f$ can always be written in the following form:

$$
f=C^{2}(\rho, \theta) \Delta(\rho) .
$$

In four dimensions the black hole has at most two horizons (e.g. see [1]) and $\Delta=(\rho-$ $\left.r_{+}\right)\left(\rho-r_{-}\right)$. When there exist more than two horizons, we call the outermost two horizons as $r_{-}, r_{+}\left(r_{+}>r_{-}\right)$. The constants $r_{+}, r_{-}$are two parameters characterizing the black hole. We introduce $r_{h}, \epsilon$ instead of $r_{ \pm}$as:

$$
r_{h} \equiv\left(r_{+}+r_{-}\right) / 2, \quad \epsilon \equiv\left(r_{+}-r_{-}\right) / 2
$$

The above notation turns out to be useful since $\epsilon$ is a good measure of black hole temperature $T_{H}$. Hawking temperature of the black hole can be found requiring the near horizon metric in the Euclidean sector to be free of conical singularity (this seems to be a well known fact, e.g. see [31]), leading to [32, 33]

$$
T_{H}=\left.\frac{1}{2 \pi} \sqrt{g^{\rho \rho}} \partial_{\rho} \sqrt{f}\right|_{\rho=r_{+}}=\frac{C D}{4 \pi}\left(r_{+}-r_{-}\right)=\frac{C D}{2 \pi} \epsilon,
$$

where in the above $C$ and $D$ are computed at the horizon $\rho=r_{+}$. Constancy of Hawking temperature on the horizon implies that $C\left(r_{+}, \theta\right) D\left(r_{+}, \theta\right)$ is a constant on the horizon [32, 33]. In the extremal limit, $\epsilon \rightarrow 0$ and $\Delta$ in (5.2) will have a double root at $\rho=r_{e}$. 


\subsection{Near horizon limit of extremal black holes}

From now on we will focus on the extremal case, $r_{+}=r_{-}=r_{e}$. To take the near horizon limit let us first make the coordinate and gauge transformations

$$
\begin{aligned}
\rho & =r_{e}(1+\lambda r), \quad \tau & =\frac{\alpha r_{e} t}{\lambda} & \\
\varphi^{i} & =\psi^{i}-\Omega^{i} \tau, \quad A^{(p)} & =\tilde{A}^{(p)}+d \Lambda, & \Lambda=-\left.\Phi^{(p)}\right|_{r_{e}} \tau
\end{aligned}
$$

where $\Omega^{i}=\omega^{i}\left(r_{e}\right)$ is the horizon angular velocity and $\left.\Phi^{(p)}\right|_{r_{e}}$ is the horizon electric potential. In the first line we scale $\rho-r_{e}$ and $\tau$ inversely by a factor $\lambda$ and $\alpha$ is a suitable constant to get the most simple form for the near horizon metric. $\lambda$ is the parameter which we send to zero once we take the limit. The shift in $\psi^{i}$ takes us to the frame co-rotating with the black hole. In the last equation, we have used the gauge symmetry in order to remove the infinities resulting from the limit $\lambda \rightarrow 0$. Upon these transformations the near horizon geometry (obtained in the $\lambda \rightarrow 0$ limit) becomes

$$
d s^{2}=\frac{1}{D^{2}}\left[-r^{2} d t^{2}+\frac{d r^{2}}{r^{2}}+D^{2} \tilde{g}_{\alpha \beta} d \theta^{\alpha} d \theta^{\beta}+D^{2} g_{i j}\left(d \varphi^{i}+\left(\Omega^{i}-\omega^{i}\right) d \tau\right)\left(d \varphi^{j}+\left(\Omega^{j}-\omega^{j}\right) d \tau\right)\right],
$$

where we used the fact that $C D=$ const on the horizon and chose

$$
\alpha r_{e}^{2}=\frac{1}{C D}
$$

Recalling that $\Omega^{i}=\left.\omega^{i}\right|_{r_{e}}$, we arrive at the general form:

$$
\begin{aligned}
& d s^{2}=\Gamma\left[-r^{2} d t^{2}+\frac{d r^{2}}{r^{2}}+g_{\alpha \beta} d \theta^{\alpha} d \theta^{\beta}+\gamma_{i j}\left(d \varphi^{i}+k^{i} r d t\right)\left(d \varphi^{j}+k^{j} r d t\right)\right] \\
& A^{(p)}=e^{(p)} r d t+\sum_{i} \mu_{i}^{(p)}\left(d \varphi^{i}+k^{i} r d t\right),
\end{aligned}
$$

in which

$$
\Gamma=\left.\frac{1}{D^{2}}\right|_{\rho=r_{e}}, \quad \gamma_{i j}=\left.D^{2} g_{i j}\right|_{\rho=r_{e}}, \quad k^{i}=-\left.\frac{1}{C D} \frac{\partial \omega^{i}}{\partial \rho}\right|_{\rho=r_{e}}, \quad e^{(p)}=\left.\frac{1}{C D} \frac{\partial \Phi^{(p)}}{\partial \rho}\right|_{\rho=r_{e}} .
$$

The above is, as expected, the same as the NHEG ansatz (2.3) and (2.4).

We first show that smoothness of black hole geometry (5.1) forces $\partial_{\rho} \omega^{i}$ to be constant on the horizon, and $k^{i}$ are hence constants in the NHEG. A more detailed proof for this has appeared in [34] (see the appendix there). However, here we give an alternative argument. Analysis of finiteness of curvature invariants for solutions to field equations of the form (5.10) reveals that $\left(\partial_{\theta^{\alpha}} \omega^{i}\right)^{2} \sim\left(\rho-r_{e}\right)^{2 \alpha}$, with $\alpha>1$. Therefore, $\left.\partial_{\rho} \partial_{\theta^{\alpha}} \omega^{i}\right|_{\rho=r_{e}}=\left.\partial_{\theta \alpha} \partial_{\rho} \omega^{i}\right|_{\rho=r_{e}}=0$. So, not only $\partial_{\theta^{\alpha}} \omega^{i}=0$ on the horizon (which means that angular velocity is constant on the horizon), but also $\partial_{\rho} \partial_{\theta^{\alpha}} \omega^{i}=0$ which means that $\partial_{\rho} \omega^{i}$ is constant at the horizon of extremal black holes. Using the third equation of (5.12), we find that $k^{i}$ are $\theta$ independent and hence constants. This is a restatement of the zeroth law for NHEG geometries (cf. section 4.1). 


\subsection{NHEG entropy perturbation law and near horizon limit}

Here we briefly review what was done in [29] (see also [30, 35, 36]): one can indeed derive "entropy variation law" of NHEG from taking the extremal limit, starting from first law of thermodynamics for near extremal black holes. To this end, we recall the first law of black holes stating how perturbation of entropy is related to the perturbations of mass and other conserved charges of any black hole:

$$
\delta M=T_{H} \delta S+\sum_{i} \Omega^{i} \delta J_{i}+\sum_{p} \Phi^{p} \delta q_{p} .
$$

At the extremal point where $T_{H}=0$ the above reduces to $\delta M=\sum_{i} \Omega^{i} \delta J_{i}+\sum_{p} \Phi^{p} \delta q_{p}$, which may in principle be integrated to get the BPS relation $M=M\left(J_{i}, q_{p}\right)$. In the near extremal case when $T_{H} \sim \epsilon$, one may then make a low temperature expansion of all thermodynamics quantities in powers of $\epsilon$. For black holes, we have the crucial relation that [29] $\delta M-\Omega_{e x t}^{i} \delta J_{i}-\Phi_{e x t}^{p} \delta q_{p} \sim \epsilon^{2}$, and hence to the leading order in $\epsilon$ the first law reduces to

$$
\delta S=-\sum_{i} \Omega^{\prime i} \delta J_{i}+\sum_{p} \Phi^{\prime p} \delta q_{p}
$$

where

$$
\Omega^{\prime i}=\left.\frac{\partial \Omega^{i}}{\partial T_{H}}\right|_{T_{H}=0}, \quad \Phi^{\prime p}=\left.\frac{\partial \Phi^{p}}{\partial T_{H}}\right|_{T_{H}=0} .
$$

eq. (5.14) reduces to the NHEG entropy perturbation law (4.5), if we show that $k^{i}=$ $-\frac{1}{2 \pi} \frac{\partial \Omega^{i}}{\partial T_{H}}, e^{p}=\frac{1}{2 \pi} \frac{\partial \Phi^{p}}{\partial T_{H}}$. That is what we will do next.

\subsection{Interpretation of $k^{i}, e^{p}$}

To relate $\Omega^{\prime i}$ and $\Phi^{\prime p}$ (which are constructed from thermodynamic chemical potential of black holes in the extremal limit) to the $k^{i}$ and $e^{p}$ which are parameters appearing in the NHEG, after taking the near horizon limit, we need to make a connection between process of taking the near extremal limit and the near horizon limit performed in section 5.1. Explicitly, we need to relate spatial derivatives of $\omega^{i}$ to the derivative of $\Omega^{i}$ (which is $\omega^{i}$ computed at the horizon) with respect to temperature. ( $\omega^{i}$ are defined in (5.1).) Similar arguments may also be repeated for the electric charges and the corresponding potentials. To do so, we use the values of the chemical potentials at inner and outer horizons and the corresponding continuity conditions.

Any function in the black hole solution (like metric components) has a spacetime and a parametric dependence. Here we choose $T_{H}$ and the conserved charges $J_{i}, q_{p}$ as the basis for parameter space of a generic black hole; the subspace $T_{H}=0$ specifies the extremal black holes. In order to relate $\partial_{\rho} \omega$ and thermodynamic quantities of black hole, we use a novel symmetry of black holes pointed out in [37] based on ideas initiated in [38-40]. We call it horizons permutation symmetry (see appendix C for a proof) which states that 
under $r_{+} \leftrightarrow r_{-}$,

$$
\begin{aligned}
& \Omega_{+}^{i} \longleftrightarrow \Omega_{-}^{i}, \\
& \Phi_{+}^{p} \longleftrightarrow \Phi_{-}^{p}, \\
& \kappa_{+} \longleftrightarrow-\kappa_{-},
\end{aligned}
$$

where $\Omega_{ \pm}^{i}, \Phi_{ \pm}^{p}, \kappa_{ \pm}$are respectively the angular velocity, horizon area and surface gravity of outer/inner horizons. This symmetry takes a more convenient form in terms of $r_{h}, \epsilon$ defined in (5.4), as

$$
r_{ \pm}=r_{h}\left(T_{H}, J, \ldots\right) \pm \epsilon
$$

Since for small $\epsilon$ temperature is proportional to $\epsilon, r_{h}=r_{h}(\epsilon, J, \ldots)$, and $r_{h} \rightarrow r_{e}$ as we take $\epsilon \rightarrow 0$. As the first step we prove that corrections to $r_{h}$ as we move away from $r_{e}$ grow like $\epsilon^{2}$ in the leading order.

Proof. We first note that $r_{h}=\left(r_{+}+r_{-}\right) / 2$ is symmetric under $r_{+} \longleftrightarrow r_{-}$, while $2 \epsilon=$ $r_{+}-r_{-}$is antisymmetric. This in particular implies that $r_{+} \longleftrightarrow r_{-}$transformation takes $\epsilon \longleftrightarrow-\epsilon$ or $T_{H} \longleftrightarrow-T_{H}$ transformation. Therefore, $r_{h}(\epsilon)=r_{h}(-\epsilon)$ and $\frac{\partial r_{h}}{\partial \epsilon}=0$ or $r_{h}=r_{e}+\mathcal{O}\left(\epsilon^{2}\right)$.

We should comment that in the above analysis, we started with $T_{H} \geq 0$ but extended the parameter space of black holes to the negative $T_{H}$ as well. The point $\left(-T_{H}, J\right)$ describes the inner horizon of the black hole with $\left(T_{H}, J\right)$ and the transformation $T_{H} \rightarrow-T_{H}$ reveals the inner horizon thermodynamics [37]. From the black hole geometry viewpoint, this is equivalent to moving from $r_{+}$to $r_{-}$and hence we have built the connection between moving in the radial direction in spacetime and moving in the parameter space of black holes, from which we can deduce our desired relations.

We now prove that radial derivative of $\omega^{i}(\rho)=g^{i j} g_{t j}$ can be related to the parametric derivative of horizon angular velocity $\Omega_{ \pm}^{i}$ w.r.t. temperature, i.e.

$$
\left.\frac{\partial \omega^{i}}{\partial \rho}\right|_{\rho=r_{e}}= \pm\left.\frac{\partial \Omega_{ \pm}^{i}}{\partial \epsilon}\right|_{\epsilon=0} .
$$

Proof. The $r_{+} \rightarrow r_{-} \Rightarrow \Omega_{+}^{i} \rightarrow \Omega_{-}^{i}$ symmetry, in the lowest order in $\epsilon$ yields

$$
\Omega_{+}^{i}-2 \epsilon \frac{\partial \Omega^{i}}{\partial \epsilon}=\Omega_{-}^{i} \Rightarrow \Omega_{+}^{i}-\Omega_{-}^{i}=2 \epsilon \frac{\partial \Omega^{i}}{\partial \epsilon}
$$

where $\Omega^{i}$ is the (outer) horizon angular velocity $\Omega_{+}^{i}$. On the other hand, by definition of $\Omega^{i}$ we have

$$
\Omega_{ \pm}^{i}=\omega^{i}\left(r_{ \pm} ; J, \epsilon\right) \Rightarrow \Omega_{+}^{i}-\Omega_{-}^{i}=\left.2 \epsilon \frac{\partial \omega^{i}}{\partial \rho}\right|_{\rho=r_{e}}
$$

and hence

$$
\left.\frac{\partial \omega^{i}}{\partial \rho}\right|_{\rho=r_{e}}=\left.\frac{\partial \Omega^{i}}{\partial \epsilon}\right|_{\epsilon=0}
$$


Similarly one can show that

$$
\left.\frac{\partial \Phi^{(p)}(\rho)}{\partial \rho}\right|_{\rho=r_{e}}=\left.\frac{\partial \Phi^{(p)}}{\partial \epsilon}\right|_{\epsilon=0} .
$$

This is an interesting identity because $\partial \omega / \partial \rho$ is completely geometrical and concerns the change of $\omega$ by moving outside the horizon of an extremal black hole, but $\partial \Omega / \partial \epsilon$ is a quantity in the parameter space and measures the change of angular velocity by turning the temperature on, and has no geometrical meaning.

We can now compute $k^{i}$ in $(5.12)$ :

$$
k^{i}=-\left.\frac{1}{C D} \frac{\partial \omega}{\partial \rho}\right|_{\rho=r_{e}}=\left.\frac{1}{2 \pi} \frac{\partial \Omega^{i}}{\partial T_{H}}\right|_{\epsilon=0},
$$

where we used (5.5). One may similarly work out $e^{p}$, and with these in hand (5.14) takes the form

$$
\delta S=2 \pi\left(\sum_{i} k^{i} \delta J_{i}+\sum_{p} e^{p} \delta q_{p}\right) .
$$

That is, we have obtained NHEG entropy perturbation law as the appropriate near extremal limit of the first law of black hole thermodynamics.

\section{Concluding remarks}

In this work we focused on the NHEG as a well-studied and classified solution to gravity theories and worked out universal relations among the parameters defining these solutions and the corresponding conserved charges. In particular we pointed out three laws of NHEG dynamics: (1) $k^{i}$ and $e^{p}$ parameters defining the NHEG are constants. (2) We have the "entropy law" which relates entropy (as a Noether charge) associated with the NHEG to conserved charges angular momenta $J^{i}$ and the electric charges $q^{p}$ and the on-shell value of Lagrangian (integrated over $H$ ), and (3) the "entropy perturbation law," which relates entropy and other Noether charges associated with a probe (probing the NHEG background) to each other.

The entropy and entropy perturbation laws, despite the similarity to laws of black hole thermodynamics do not indeed have a thermodynamical interpretation; in the NHEG case we are dealing with a system which cannot be excited (without destroying the $\mathrm{SL}(2, \mathbb{R}$ ) isometry) $[26,27,29]$. Among other points, we would like to stress that the entropy law does not have a correspondent in the black hole thermodynamics systems. Technically, this is due to the fact that in the Wald's derivation of the first law for black holes there are ambiguities defining the charge integrals which prevents one to draw a universal relation among the thermodynamical parameters of black holes, while such ambiguities does vanish when we consider variations of fields and the corresponding perturbations in the thermodynamical charges, as they appear in the first law of thermodynamics. 
It is worth also mentioning that the entropy and entropy perturbation laws are invariant under permutation of $N \mathrm{U}(1)$ symmetries. Under these permutations $k^{i}$ and $e^{p}$ and the corresponding charges are rotated into each other, while $S$ and $\delta S$ are only a function invariant under these permutations. It is interesting to explore this permutation symmetry further.

Regarding the entropy perturbation law, as we discussed $\delta S, \delta J_{i}$ and $\delta q_{p}$ are associated with a field configuration $\delta \Phi$ probing the NHEG background, given by the field configuration $\Phi_{0}$. As we argued, entropy perturbation law (4.5) is valid for $\delta \Phi$ satisfying equations of motion linearized around background $\Phi_{0}$. Moreover, $\delta \Phi$ should be such that $\delta \mathcal{E}_{a}=0$. Given the discussions in $[26,27]$ one may wonder if these two conditions can be satisfied. Our preliminary analysis [28] shows the answer is positive. In answering this question one may also explore if there is any relation between these $\delta \Phi$ and the set of perturbations and boundary conditions appearing in the Kerr/CFT proposal [35, 41]. It is also desirable to understand better the connection of our derivations and the NHEG mechanics with the entropy function analysis. This is also postponed to future works.

In general, especially when we deal (extremal) black holes of non-trivial horizon topology, it is possible to have solutions with non-zero "dipole charges". One such example is the neutral singly rotating dipole black ring [24]. The dipole charge in fact contributes to the energy of the system and appears both in first law or the Smarr-type relation for the dipole black ring [24]. Following Wald's derivation for the first law one can in fact prove that in general such dipole charges should appear in the first law [25]. In principle black holes/rings with dipole charges can become extremal. For example the five dimensional dipole black ring of [24] can become extremal while the dipole charge is still non-zero. One may study near horizon limit of extremal dipole rings and see that they exhibit $\mathrm{SL}(2, \mathbb{R}) \times \mathrm{U}(1)^{2}[16]$ and hence they fall into our definition of the NHEG. One then expects these dipole charges to appear both in our entropy law and in the entropy perturbation law [28].

One may wonder if the second law of thermodynamics has a correspondent in the NHEG case. Here we make a comment on that and postpone a more thorough analysis to the future publications. Let us for simplicity consider the NHEG ansatz (2.3). One may show that the angular momentum $J_{i}$ is given by the Noether integration

$$
J_{i} \propto \int_{H} F(\theta) \gamma_{i j} k^{j} \quad \Longrightarrow \quad k^{i} J_{i} \propto \int_{H} F(\theta) k^{i} \gamma_{i j} k^{j}
$$

where $F(\theta)$ is a positive definite function and $\gamma_{i j}$ is also a positive definite metric on the $\phi^{i}$ part of the NHEG geometry. Therefore, $k^{i} J_{i}$ is positive definite. Similar relation also holds for $e^{p} q_{p}$.

We also discussed a derivation of NHEG mechanics laws from near extremal black holes, this latter amount to finding a relation between spatial derivatives of black hole metric functions and the parametric derivatives of the chemical potentials (horizon angular velocities or electric potentials). To this end we proved and used the inner-outer horizon exchange symmetry (see discussions in section 5 and appendix D). It is desirable to understand this symmetry better and study its further implications. 


\section{Acknowledgments}

We would like to thank Bin Chen, Geoffrey Compère, Monica Guica, Finn Larsen, Saeedeh Sadeghian, Joan Simón, Hossein Yavartanoo, Jia-ju Zhang for fruitful discussions and/or comments on the draft. We would like to also thank the anonymous referee for very useful comments which helped us improving the presentation of our results. KH and AS would like to thank Mehdi Golshani for his encouragement and guidance. MMShJ would like to thank organizers of "Open Questions in Open Universe" workshop, held in August 2013 in Istanbul, where preliminary results of this work was presented. We would also like to thank organizers of workshop "Quantum Aspects of Black Holes and its Recent Progress", held in Yerevan, September 2013, where this work was presented.

\section{A On $\operatorname{sl}(2, \mathbb{R})$ Lie algebra}

$\operatorname{SL}(2, \mathbb{R})$ is the group of all $2 \times 2$ real-valued matrices with determinant one. The $\operatorname{sl}(2, \mathbb{R})$ Lie algebra with generators $\xi_{a}, a=1,2,3$ is defined as

$$
\left[\xi_{a}, \xi_{b}\right]=f_{a b}^{c} \xi_{c}
$$

where $f_{a b}{ }^{c}$ are structure constants. In this paper we have chosen the basis in a way that the commutation relations take the form

$$
\left[\xi_{1}, \xi_{2}\right]=\xi_{1}, \quad\left[\xi_{2}, \xi_{3}\right]=\xi_{3}, \quad\left[\xi_{1}, \xi_{3}\right]=\xi_{2} .
$$

In this basis, the Killing form (metric) of the algebra is

$$
K_{a b}=\left(\begin{array}{ccc}
0 & 0 & 1 \\
0 & -1 & 0 \\
1 & 0 & 0
\end{array}\right)
$$

and its inverse $K^{a b}=\left(K_{a b}\right)^{-1}$ has the same components as itself (in the chosen basis). Metric $K_{a b}$ can be used for lowering or raising the $s l(2, \mathbb{R})$ indices, e.g. $f_{a b c}=K_{c d} f_{a b}{ }^{d}$. One may also show that

$$
f_{a b}^{c} f^{a b d}=2 K^{c d} .
$$

One specific representation of the $\operatorname{sl}(2, \mathbb{R})$ algebra, which also realized the $\operatorname{SL}(2, \mathbb{R})$ isometry of $(2.3)$, is given in $(2.5)$.

$\mathrm{SL}(2, \mathbb{R})$ which is a double cover of $\mathrm{SO}(2,1)$ is also the isometry group of $\mathrm{AdS}_{2}$ manifold, defined as the set of points with square distance -1 from the origin of a flat $1+2$ dimensional Minkowski space. In a suitable coordinate system in which the metric is (A.3), this condition is explicitly

$$
n^{a} n_{a}=K^{a b} n_{a} n_{b}=-1,
$$

where $x_{a}=n_{a}$ are the position of points of $\mathrm{AdS}_{2}$ in the embedding space. coordinates. A solution for $n_{a}$, parametrized with two parameters $t, r$ is

$$
n_{1}=-r, \quad n_{2}=-t r, \quad n_{3}=-\frac{t^{2} r^{2}-1}{2 r},
$$


then the induced metric on the $\mathrm{AdS}_{2}$ surface is

$$
d s^{2}=-r^{2} d t^{2}+\frac{d r^{2}}{r^{2}}
$$

which is the metric of $\mathrm{AdS}_{2}$ in Poincaré patch. The $n_{a}, a=1,2,3$ form a vector representation under $\mathrm{SL}(2, \mathbb{R})$ and hence,

$$
\delta_{\xi_{a}} n_{b}=f_{a b}^{c} n_{c}, \quad \delta_{\xi_{a}}\left(n_{b} n^{b}\right)=0,
$$

where $\delta_{\xi_{a}} n_{b}$ is the Lie derivative of the vector $n_{b}$. Using the explicit form of (2.5) and (2.9) one may show that

$$
n^{a} \delta_{\xi_{a}} n_{b}=0, \quad \delta_{\xi_{a}} n_{b}=\xi_{a}^{t} \xi_{b}^{r}-\xi_{a}^{r} \xi_{b}^{t} .
$$

The above relations also show that the constant $r, t$ part of the NHEG metric (2.3), the codimension two surface $H$, is an $\operatorname{SL}(2, \mathbb{R})$ invariant space, i.e. its metric and volume form do not depend on which constant $r, t$ the surface $H$ is defined.

Definition. The binormal tensor of the $\mathrm{SL}(2, \mathbb{R})$ invariant surfaces $H$ is defined as:

$$
\epsilon_{\mu \nu} \equiv \xi_{\mu}^{a} \nabla_{\nu} n_{a}
$$

In the basis (2.5) and coordinate (2.9), this tensor can be calculated as follows:

$$
\epsilon_{\mu \nu}=\xi_{a \mu} \partial_{\nu} n^{a}=K^{a b} \xi_{a \mu} \partial_{\nu} n_{b}=K^{a b} \xi_{a \mu}\left(\delta_{\nu}^{t} \xi_{b}^{r}-\delta_{\nu}^{r} \xi_{b}^{t}\right),
$$

where in the last equality we used $\partial_{r} n_{a}=-\xi_{a}^{t}, \partial_{t} n_{a}=\xi_{a}^{r}$. Explicit computation for $\mu=r, t$ and with metric (2.3) yields

$$
K^{a b} \xi_{a r} \xi_{b}^{r}=K^{a b} \xi_{a t} \xi_{b}^{t}=-\Gamma,
$$

and zero for the other components. The final result is that

$$
\epsilon_{\mu \nu}=\left\{\begin{array}{l}
\epsilon_{t r}=-\epsilon_{r t}=\Gamma \\
0 \quad \text { other components }
\end{array}\right.
$$

or as a 2 -form

$$
\epsilon=\Gamma d t \wedge d r=\frac{1}{\sqrt{-g^{t t} g r^{r r}}} d t \wedge d r
$$

One can also readily show that

$$
\epsilon^{2} \equiv \epsilon_{\mu \nu} \epsilon^{\mu \nu}=-2
$$

\section{A.1 $\mathrm{AdS}_{2}$ in global coordinates, another example}

As another example, let us consider NHEG in the global coordinate for $\mathrm{AdS}_{2}$ :

$d s^{2}=\Gamma\left[-\left(1+r^{2}\right) d t^{2}+\frac{d r^{2}}{1+r^{2}}+\sum_{\alpha, \beta=1}^{d-n-2} \Theta_{\alpha \beta} d \theta^{\alpha} d \theta^{\beta}+\sum_{i, j=1}^{n} \gamma_{i j}\left(d \varphi^{i}+k^{i} r d t\right)\left(d \varphi^{j}+k^{j} r d t\right)\right]$ 
where $\Gamma, \Theta_{\alpha \beta}, \gamma_{i j}$ are some functions of $\theta^{\alpha}$, specified by the equations of motion. Associated with this coordinate system, the $\mathrm{sl}(2, \mathbb{R})$ Killing vector fields are given as

$$
\begin{aligned}
& \xi_{1}=\partial_{t}, \\
& \xi_{2}=\sin t \frac{r}{\sqrt{1+r^{2}}} \partial_{t}-\cos t \sqrt{1+r^{2}} \partial_{r}+\sin t \sum_{i=1}^{n} \frac{k^{i}}{\sqrt{1+r^{2}}} \partial_{\varphi^{i}}, \\
& \xi_{3}=-\cos t \frac{r}{\sqrt{1+r^{2}}} \partial_{t}-\sin t \sqrt{1+r^{2}} \partial_{r}-\cos t \sum_{i=1}^{n} \frac{k^{i}}{\sqrt{1+r^{2}}} \partial_{\varphi^{i}} .
\end{aligned}
$$

In this basis the $\operatorname{sl}(2, \mathbb{R})$ commutation relations and metric are

$$
\begin{aligned}
{\left[\xi_{1}, \xi_{2}\right] } & =-\xi_{3}, \quad\left[\xi_{3}, \xi_{1}\right]=-\xi_{2}, \quad\left[\xi_{2}, \xi_{3}\right]=\xi_{1} \\
K_{a b} & =\left(\begin{array}{ccc}
1 & 0 & 0 \\
0 & -1 & 0 \\
0 & 0 & -1
\end{array}\right)
\end{aligned}
$$

The solution to (A.5) which also satisfies (A.8) is now given as

$$
n_{1}=-r, \quad n_{2}=-\sqrt{1+r^{2}} \sin t, \quad n_{3}=\sqrt{1+r^{2}} \cos t .
$$

It can be checked that relations $\partial_{r} n_{a}=-\xi_{a}^{t}$ and $\partial_{t} n_{a}=\xi_{a}^{r}$ also hold in the global coordinate and hence (A.9) is still true. Using the same discussion as above one can show that using the definition (A.10) leads to the same result for the binormal tensor

$$
\epsilon_{\mu \nu}=\frac{1}{\sqrt{-g^{t t} g^{r r}}} d t \wedge d r
$$

\section{B Symmetries and conserved charges}

Symmetry is a transformation which maps a set of solutions of equations of motion (with appropriate boundary conditions) to themselves and hence leaves the action invariant, or equivalently, changes the Lagrangian up to a total divergence. The symmetries could be local (gauge) or global and both of these have been argued to be a basis for deriving constants of motion or conserved charges, see [42] and references therein for a historical review. Here we will be mainly concerned with symmetries associated with spacetime coordinate transformations and diffeomorphisms and will follow Wald's papers [9, 10, 12].

Consider a diffeomorphism invariant theory with a Lagrangian density $\mathcal{L}$ and the corresponding action in $d$-dimensional space-time

$$
I[\phi]=\int \mathrm{d}^{d} x \sqrt{-g} \mathcal{L}\left(\Phi ; x^{\mu}\right)
$$

in which $\Phi$ denotes all of dynamical fields of the system and each of them will be denoted by $\Phi^{i}$. Associated with any infinitesimal diffeomorphism as a symmetry of the theory, one can find a Noether current and the corresponding Noether charge. Following [10] we take 
the Lagrangian $\mathbf{L}$ to be a top form, a $d$-form equal to $\sqrt{-g} \mathcal{L} \epsilon_{d}$ with $\epsilon_{d}$ being the LeviCivita tensor, and generator of diffeomorphism symmetry to be a 1-form $\xi$. Variation of Lagrangian under the diffeomorphism is [43]

$$
\delta_{\xi} \mathbf{L}=\mathbf{E}_{i} \delta_{\xi} \Phi^{i}+\mathrm{d} \boldsymbol{\Theta}\left(\Phi, \delta_{\xi} \Phi\right)
$$

where $\mathbf{E}_{i}=0$ is the e.o.m for $\Phi^{i}$. The $(d-1)$-form $\boldsymbol{\Theta}$ is the surface term generated by the variation.

According to the identity $\delta_{\xi} \mathbf{L}=\xi \cdot \mathrm{d} \mathbf{L}+\mathrm{d}(\xi \cdot \mathbf{L})$ and noting that $\mathrm{d} \mathbf{L}=0$, we can replace the 1.h.s. of (B.2):

$$
\mathrm{d} \boldsymbol{\Theta}\left(\Phi, \delta_{\xi} \Phi\right)-\mathrm{d}(\xi \cdot \mathbf{L})=-\mathbf{E}_{i} \delta_{\xi} \Phi^{i}
$$

Now, we can associate a Noether $(d-1)$-form current $\mathbf{J}$ as:

$$
\mathbf{J} \equiv \boldsymbol{\Theta}\left(\Phi, \delta_{\xi} \Phi\right)-\xi \cdot \mathbf{L}
$$

Therfore $\mathrm{d} \mathbf{J}=-\mathbf{E}_{i} \delta_{\epsilon} \Phi^{i}$ so that $\mathrm{d} \mathbf{J}=0$ whenever e.o.m is satisfied and according to the Poincaré's lemma, since $\mathbf{J}$ is closed, it would be exact and can be written as:

$$
\mathbf{J}=\mathrm{d} \mathbf{Q}
$$

where $\mathbf{Q}$ is a $(d-2)$-form, the Noether charge density.

\section{B.1 Ambiguities}

It has been shown $[10,12]$ that the $(d-1)$-form $\mathbf{J}$ in (B.4) has twofold ambiguities. One ambiguity comes from freedom of the definition of Lagrangian of the theory up to an exact $d$-form:

$$
\mathcal{L} \rightarrow \mathcal{L}+d \mu,
$$

which leads to $\mathbf{J} \rightarrow \mathbf{J}+\delta_{\xi} \mu$. The other ambiguity comes from the freedom in specifying $\mathbf{J}$ itself (for a given Lagrangian) up to an exact $(d-1)$-form $d Y(\Phi, \delta \Phi)$. Therefore, the Noether current $\mathbf{J}$ is defined up to the following ambiguities

$$
\mathbf{J} \rightarrow \mathbf{J}+d(\xi \cdot \mu)+d Y(\Phi, \delta \Phi),
$$

where the $(d-2)$-form $Y(\Phi, \delta \Phi)$ is linear in $\delta_{\xi} \Phi$ and we used the identity $\delta_{\xi} \mu=\xi \cdot d \mu+d(\xi \cdot \mu)$. When we want to find the Noether charge, in addition to these ambiguities there is another one which is the freedom of choosing $\mathbf{Q}$ up to an exact $(d-2)$-form $d Z(\Phi, \xi)$ where $Z$ is linear in $\xi$. So accumulating all of the ambiguities, we have the freedom of choosing the Noether charge density as:

$$
\mathbf{Q} \rightarrow \mathbf{Q}+\xi \cdot \mu+Y+d Z
$$

and hence the Noether charge density $\mathbf{Q}$ is not unique and its most general is [10]

$$
\mathbf{Q}=W_{\mu}(\Phi) \xi^{\mu}+\mathbf{E}^{\mu \nu}(\Phi) \nabla_{[\mu} \xi_{\nu]}+Y\left(\Phi, \delta_{\xi} \Phi\right)+d Z(\Phi, \xi)
$$


where $W_{\mu}$ and $\mathbf{E}^{\mu \nu}$ and $Y$ and $Z$ are covariant quantities which are locally constructed from fields and their derivatives, $Y$ is linear in $\delta_{\xi} \Phi, Z$ is linear in $\xi$ and,

$$
\left(\mathbf{E}^{\mu \nu}\right)_{\alpha_{3} \ldots \alpha_{d}}=-E^{\alpha \beta \mu \nu} \epsilon_{\alpha \beta \alpha_{3} \ldots \alpha_{d}}, \quad E^{\mu \nu \alpha \beta} \equiv \frac{\delta \mathcal{L}}{\delta R_{\mu \nu \alpha \beta}} .
$$

In order to fix/remove these ambiguities, we need some physical reasoning and/or reference point for defining the charges (like requesting to coincide with the ADM charges etc.)

\section{B.2 Iyer-Wald entropy}

Iyer-Wald entropy $[9,10]$ for a generic stationary black hole with bifurcate horizon is defined as:

$$
\frac{S}{2 \pi} \equiv-\int_{\mathcal{H}} \operatorname{Vol}(\mathcal{H}) \frac{\delta \mathcal{L}}{\delta R_{\mu \nu \alpha \beta}} \epsilon_{\mu \nu} \epsilon_{\alpha \beta}
$$

where $\epsilon_{\alpha \beta}=n_{[\alpha} \xi_{\beta]}$ is the binormal for the $d$-2-dimensional horizon surface $\mathcal{H}$ and the vectors $\xi_{\mu}$ and $n_{\mu}$ are normals to the bifurcate horizon null surface which on the horizon satisfy the relations

$$
n \cdot n=0, \quad \xi \cdot \xi=0, \quad n \cdot \xi=-1
$$

and according to them, the binormal satisfies $\epsilon^{2}=-2$.

\section{Computation of symplectic form}

Here we present details of computation of the symplectic form appearing in the l.h.s. of (4.17). As mentioned [10], the symplectic current $\boldsymbol{\omega}=0$ for $\delta_{\xi} \Phi=0$. This is true for the Killing vectors of NHEG, except for $\xi_{3}$ when acting on gauge fields where there is a residual gauge transformation. To compute the effects of this residual gauge transformation, we start with the definition of $\boldsymbol{\omega}$

$$
\boldsymbol{\omega}\left(\Phi, \delta \Phi, \delta_{\xi} \Phi\right)=\delta \boldsymbol{\Theta}\left(\delta_{\xi} \phi\right)-\delta_{\xi} \boldsymbol{\Theta}(\delta \phi) .
$$

We discussed the form of $\boldsymbol{\Theta}$, or its Hodge dual vector field $\Theta^{\mu}$, for gauge fields in (3.9):

$$
\Theta^{\mu}\left(\delta A_{\alpha}\right)=\frac{\partial \mathcal{L}}{\partial F_{\mu \nu}} \delta A_{\nu}
$$

so

$$
\begin{aligned}
\delta_{2} \Theta^{\mu}\left(\delta_{1} A_{\alpha}\right) & =\delta_{2}\left(\frac{\partial \mathcal{L}}{\partial F_{\mu \nu}} \delta_{1} A_{\nu}\right) \\
& =\delta_{2}\left(\frac{\partial \mathcal{L}}{\partial F_{\mu \nu}}\right) \delta_{1} A_{\nu}+\frac{\partial \mathcal{L}}{\partial F_{\mu \nu}} \delta_{2} \delta_{1} A_{\nu} .
\end{aligned}
$$

Assuming that $\delta_{1} \delta_{2}=\delta_{2} \delta_{1}$ (which is true for $\delta, \delta_{\xi}$ )

$$
\omega^{\mu}\left(\Phi, \delta_{1} \Phi, \delta_{2} \Phi\right)=\delta_{2}\left(\frac{\partial L}{\partial F_{\mu \nu}}\right) \delta_{1} A_{\nu}-\delta_{1}\left(\frac{\partial L}{\partial F_{\mu \nu}}\right) \delta_{2} A_{\nu}
$$


where $\omega^{\mu}$ is the vector Hodge dual to the $(d-1)$-form symplectic current $\boldsymbol{\omega}$. The nonvanishing part of $\omega$ is hence

$$
\begin{aligned}
\omega^{\mu}\left(\Phi, \delta \Phi, \delta_{\xi_{3}} \Phi\right) & =\delta\left(\frac{\partial L}{\partial F_{\mu \nu}}\right) \delta_{\xi_{3}} A_{\nu}-\delta_{\xi_{3}}\left(\frac{\partial L}{\partial F_{\mu \nu}}\right) \delta A_{\nu} \\
& =\delta\left(\frac{\partial L}{\partial F_{\mu \nu}}\right) \delta_{\xi_{3}} A_{\nu} .
\end{aligned}
$$

The second term on the right hand side is zero since $\xi_{3}$ is a symmetry of Lagrangian and $F_{\mu \nu}$. Next, recall from (3.13) that

$$
\delta_{\xi_{3}} A_{\nu}=\left(0,-\frac{e}{r^{2}}, 0,0\right)=\nabla_{\nu} \Lambda, \quad \Lambda=\frac{e}{r}
$$

therefore

$$
\begin{aligned}
\omega^{\mu}\left(\Phi, \delta \Phi, \delta_{\xi_{3}} \Phi\right) & =\delta\left(\frac{\partial L}{\partial F_{\mu \nu}}\right) \nabla_{\nu} \Lambda \\
& =\nabla_{\nu}\left(\Lambda \delta\left(\frac{\partial L}{\partial F_{\mu \nu}}\right)\right)-\Lambda \nabla_{\nu} \delta\left(\frac{\partial L}{\partial F_{\mu \nu}}\right) \\
& =\nabla_{\nu}\left(\Lambda \delta\left(\frac{\partial L}{\partial F_{\mu \nu}}\right)\right)
\end{aligned}
$$

where we have used the linearized equation of motion for the gauge field perturbations $\delta A_{\mu}$. Therefore, we obtain

$$
\begin{aligned}
\Omega\left(\Phi, \delta \Phi, \delta_{\xi_{3}} \Phi\right) & =\int_{\Sigma} d \Sigma_{\mu} \omega^{\mu}\left(\Phi, \delta \Phi, \delta_{\xi_{3}} \Phi\right)=\int_{\Sigma} d \Sigma_{\mu} \nabla_{\nu}\left(\Lambda \delta\left(\frac{\partial L}{\partial F_{\mu \nu}}\right)\right) \\
& =\oint_{\partial \Sigma} d \Sigma_{\mu \nu} \Lambda \delta\left(\frac{\partial L}{\partial F_{\mu \nu}}\right)
\end{aligned}
$$

where $\Sigma$ is a constant time slice bounded between $r=r_{H}$ and $r=\infty$. $\Omega$ will hence have a term at infinity and a term on $H$. The term at infinity does not contribute since $\Lambda=\frac{e}{r}$ vanishes there (in fact $r=\infty$ boundary was chosen precisely for this reason). So, the only contribution is

$$
\begin{aligned}
\Omega\left(\Phi, \delta \Phi, \delta_{\xi_{3}} \Phi\right) & =\oint_{H} d \Sigma_{\mu \nu}\left(\Lambda \delta\left(\frac{\partial L}{\partial F_{\mu \nu}}\right)\right) \\
& =\frac{e}{r_{H}} \delta q
\end{aligned}
$$

where $\delta q=\oint_{H} d \Sigma_{\mu \nu} \delta\left(\frac{\partial L}{\partial F_{\mu \nu}}\right)$. Noting that $\zeta_{H}=n_{H}^{a} \xi_{a}-k^{i} m_{i}$, and that $\Omega$ is linear in $\delta_{\zeta_{H}} A=n_{H}^{a} \delta_{\xi_{a}} A-k^{i} \delta_{m_{i}} A$, we obtain

$$
\Omega\left(\Phi, \delta \Phi, \delta_{\zeta_{H}} \Phi\right)=n_{H}^{3} \Omega\left(\Phi, \delta \Phi, \delta_{\xi_{3}} \Phi\right)=n_{H}^{3} \frac{e^{p}}{r_{H}} \delta q_{p}=-e^{p} \delta q_{p}
$$




\section{Inner/outer horizons permutation symmetry}

In this appendix we state and prove the permutation symmetry of black hole horizons. Permutation symmetry states that: ${ }^{7}$

Let $\left\{r_{i}\right\}$ denote the position of horizons of a given black hole, a permutation in black hole parameters of the form $r_{i} \rightarrow r_{\sigma_{i}}$, has the following effect on black hole horizon chemical potentials:

$$
\Omega_{i} \stackrel{r_{i} \rightarrow r_{\sigma_{i}}}{\longrightarrow} \Omega_{\sigma_{i}}
$$

Proof. we assume that $\Delta$ is an analytic function of $r$, then $\Delta=\sum_{m=0}^{n} c_{m} r^{m}$ which has $n$ roots $\left\{r_{i}\right\}$ and $n$ constants $c_{m}$,

$$
\Delta\left(r_{i} ;\left\{c_{m}\right\}\right)=0, \quad i=1,2, \cdots, n .
$$

If we consider $c_{m}$ 's as unknowns and $r_{i}$ as given parameters, the above is a system of linear equations which can be uniquely solved to write $c_{m}$ 's in terms of $r_{m}$ 's which results in $c_{m}=c_{m}\left(r_{1}, r_{2}, \ldots\right)$. Now a transformation of the form $\left(r_{i}\right) \rightarrow\left(r_{\sigma_{i}}\right)$ where $\sigma$ is a permutation function of $1,2, \ldots, n$, does not change the set of equations and as a result, the solutions $c_{m}=c_{m}\left(r_{1}, r_{2}, \ldots\right)$ are still solutions, and from the fact that the solution is unique, this means that $c_{m}\left(r_{\sigma(i)}\right)=c_{m}\left(r_{i}\right)$. Therefore,

$$
\Omega_{i}=\omega\left(r=r_{i} ;\left\{c_{m}\right\}\right) \stackrel{r_{i} \rightarrow r_{\sigma(i)}}{\longrightarrow} \omega\left(r=r_{\sigma(i)} ;\left\{c_{m}\right\}\right)=\Omega_{\sigma(i)} .
$$

Although $\omega$ can in principle depend on other parameters of black holes, than $c_{i}$, e.g $d_{1}, d_{2}, \ldots$ this dependence is not relevant to our argument because the transformation $r_{i} \rightarrow r_{\sigma(i)}$ does not change $d_{i}$. The reason is that we assume the system of equations (D.2) has a unique solution, and so $r_{i}$ is completely determined by $c_{m}$ 's and does not depend on $d_{m}$ 's and changing (permuting) $r_{i}$ 's does not affect $d_{m}$ 's and our argument still holds.

Open Access. This article is distributed under the terms of the Creative Commons Attribution License (CC-BY 4.0), which permits any use, distribution and reproduction in any medium, provided the original author(s) and source are credited.

\section{References}

[1] P.T. Chrusciel, J.L. Costa and M. Heusler, Stationary Black Holes: Uniqueness and Beyond, Living Rev. Rel. 15 (2012) 7 [arXiv:1205.6112] [INSPIRE].

[2] W. Israel, Event horizons in static vacuum space-times, Phys. Rev. 164 (1967) 1776 [INSPIRE].

[3] W. Israel, Event horizons in static electrovac space-times, Commun. Math. Phys. 8 (1968) 245 [INSPIRE].

[4] R. Emparan and H.S. Reall, Black Holes in Higher Dimensions, Living Rev. Rel. 11 (2008) 6 [arXiv:0801.3471] [INSPIRE].

\footnotetext{
${ }^{7}$ We thank Bin Chen and Jia-ju Zhang for correspondence on this point.
} 
[5] S.W. Hawking, Particle Creation by Black Holes, Commun. Math. Phys. 43 (1975) 199 [Erratum ibid. 46 (1976) 206-206] [INSPIRE].

[6] J.D. Bekenstein, Black holes and entropy, Phys. Rev. D 7 (1973) 2333 [inSPIRE].

[7] J.M. Bardeen, B. Carter and S.W. Hawking, The Four laws of black hole mechanics, Commun. Math. Phys. 31 (1973) 161 [InSPIRE].

[8] G.W. Gibbons, R. Kallosh and B. Kol, Moduli, scalar charges and the first law of black hole thermodynamics, Phys. Rev. Lett. 77 (1996) 4992 [hep-th/9607108] [INSPIRE].

[9] R.M. Wald, Black hole entropy is the Noether charge, Phys. Rev. D 48 (1993) 3427 [gr-qc/9307038] [INSPIRE].

[10] V. Iyer and R.M. Wald, Some properties of Noether charge and a proposal for dynamical black hole entropy, Phys. Rev. D 50 (1994) 846 [gr-qc/9403028] [INSPIRE].

[11] V. Iyer and R.M. Wald, A Comparison of Noether charge and Euclidean methods for computing the entropy of stationary black holes, Phys. Rev. D 52 (1995) 4430 [gr-qc/9503052] [INSPIRE].

[12] R.M. Wald, The thermodynamics of black holes, Living Rev. Rel. 4 (2001) 6 [gr-qc/9912119] [INSPIRE].

[13] J.M. Bardeen and G.T. Horowitz, The Extreme Kerr throat geometry: A Vacuum analog of $A d S_{2} \times S^{2}$, Phys. Rev. D 60 (1999) 104030 [hep-th/9905099] [INSPIRE].

[14] A. Sen, Black hole entropy function and the attractor mechanism in higher derivative gravity, JHEP 09 (2005) 038 [hep-th/0506177] [INSPIRE].

[15] D. Astefanesei, K. Goldstein, R.P. Jena, A. Sen and S.P. Trivedi, Rotating attractors, JHEP 10 (2006) 058 [hep-th/0606244] [INSPIRE].

[16] H.K. Kunduri, J. Lucietti and H.S. Reall, Near-horizon symmetries of extremal black holes, Class. Quant. Grav. 24 (2007) 4169 [arXiv:0705.4214] [InSPIRE].

[17] H.K. Kunduri and J. Lucietti, A Classification of near-horizon geometries of extremal vacuum black holes, J. Math. Phys. 50 (2009) 082502 [arXiv:0806.2051] [INSPIRE].

[18] H.K. Kunduri and J. Lucietti, Classification of near-horizon geometries of extremal black holes, Living Rev. Rel. 16 (2013) 8 [arXiv:1306.2517] [INSPIRE].

[19] A. Sen, Black Hole Entropy Function, Attractors and Precision Counting of Microstates, Gen. Rel. Grav. 40 (2008) 2249 [arXiv:0708.1270] [INSPIRE].

[20] S. Ferrara and R. Kallosh, Supersymmetry and attractors, Phys. Rev. D 54 (1996) 1514 [hep-th/9602136] [INSPIRE].

[21] S. Ferrara, R. Kallosh and A. Strominger, N=2 extremal black holes, Phys. Rev. D 52 (1995) 5412 [hep-th/9508072] [INSPIRE].

[22] A. Strominger, Macroscopic entropy of $N=2$ extremal black holes, Phys. Lett. B 383 (1996) 39 [hep-th/9602111] [INSPIRE].

[23] A. Sen, Quantum Entropy Function from AdS $S_{2} / C F T_{1}$ Correspondence, Int. J. Mod. Phys. A 24 (2009) 4225 [arXiv:0809.3304] [INSPIRE].

[24] R. Emparan, Rotating circular strings and infinite nonuniqueness of black rings, JHEP 03 (2004) 064 [hep-th/0402149] [INSPIRE]. 
[25] K. Copsey and G.T. Horowitz, The Role of dipole charges in black hole thermodynamics, Phys. Rev. D 73 (2006) 024015 [hep-th/0505278] [INSPIRE].

[26] O.J.C. Dias, H.S. Reall and J.E. Santos, Kerr-CFT and gravitational perturbations, JHEP 08 (2009) 101 [arXiv:0906.2380] [inSPIRE].

[27] A.J. Amsel, G.T. Horowitz, D. Marolf and M.M. Roberts, No Dynamics in the Extremal Kerr Throat, JHEP 09 (2009) 044 [arXiv: 0906. 2376] [INSPIRE].

[28] K. Hajian, A. Seraj and M.M. Sheikh-Jabbari, work in progress.

[29] M. Johnstone, M.M. Sheikh-Jabbari, J. Simon and H. Yavartanoo, Extremal Black Holes and First Law of Thermodynamics, Phys. Rev. D 88 (2013) 101503 [arXiv:1305.3157] [INSPIRE].

[30] G. Compere, The Kerr/CFT correspondence and its extensions: a comprehensive review, Living Rev. Rel. 15 (2012) 11 [arXiv:1203.3561] [INSPIRE].

[31] P.K. Townsend, Black holes: Lecture notes, gr-qc/9707012 [InSPIRE].

[32] D. Astefanesei and H. Yavartanoo, Stationary black holes and attractor mechanism, Nucl. Phys. B 794 (2008) 13 [arXiv:0706.1847] [InSPIRE].

[33] M.M. Sheikh-Jabbari and H. Yavartanoo, EVH Black Holes, AdS3 Throats and EVH/CFT Proposal, JHEP 10 (2011) 013 [arXiv:1107.5705] [InSPIRE].

[34] P. Figueras, H.K. Kunduri, J. Lucietti and M. Rangamani, Extremal vacuum black holes in higher dimensions, Phys. Rev. D 78 (2008) 044042 [arXiv:0803.2998] [INSPIRE].

[35] T. Hartman, K. Murata, T. Nishioka and A. Strominger, CFT Duals for Extreme Black Holes, JHEP 04 (2009) 019 [arXiv:0811.4393] [INSPIRE].

[36] T. Azeyanagi, G. Compere, N. Ogawa, Y. Tachikawa and S. Terashima, Higher-Derivative Corrections to the Asymptotic Virasoro Symmetry of $4 d$ Extremal Black Holes, Prog. Theor. Phys. 122 (2009) 355 [arXiv: 0903.4176] [INSPIRE].

[37] B. Chen, S.-x. Liu and J.-j. Zhang, Thermodynamics of Black Hole Horizons and Kerr/CFT Correspondence, JHEP 11 (2012) 017 [arXiv:1206. 2015] [INSPIRE].

[38] F. Larsen, A String model of black hole microstates, Phys. Rev. D 56 (1997) 1005 [hep-th/9702153] [INSPIRE].

[39] M. Cvetič and F. Larsen, Grey body factors for rotating black holes in four-dimensions, Nucl. Phys. B 506 (1997) 107 [hep-th/9706071] [INSPIRE].

[40] M. Cvetič, G.W. Gibbons and C.N. Pope, Universal Area Product Formulae for Rotating and Charged Black Holes in Four and Higher Dimensions, Phys. Rev. Lett. 106 (2011) 121301 [arXiv: 1011.0008] [INSPIRE].

[41] M. Guica, T. Hartman, W. Song and A. Strominger, The Kerr/CFT Correspondence, Phys. Rev. D 80 (2009) 124008 [arXiv:0809.4266] [INSPIRE].

[42] K.A. Brading, Which Symmetry? Noether, Weyl and Conservation of Electric Charge, Stud. His. Phil. Mod. Phys. 33 (2002) 3.

[43] J. Lee and R.M. Wald, Local symmetries and constraints, J. Math. Phys. 31 (1990) 725 [INSPIRE]. 